\title{
ESTRUCTURACIÓN DEL PROCESO DE RECEPCIÓN, ASIGNACIÓN Y EVACUACIÓN DE REQUERIMIENTOS DE TRÁMITES JURÍDICOS DE LOS CLIENTES DE LA EMPRESA VELASCO ORDÓÑEZ S.A.S. MEDIANTE LA METODOLOGÍA BUSINESS PROCESS MANAGEMENT (BPM)
}

\begin{abstract}
Structuring of the reception, assignment and requirements evacuation process, of legal processing of the clients of the company Velasco Ordónez S.A.S., through the methodology Business Process Management (BPM) ${ }^{1}$
\end{abstract}

LUISA GÁMEZ CARRACEDO², ING. ÉVER ÁNGEL FUENTES ROJAS MBA ${ }^{3}$

Recibido:20 de Septiembre de 2019. Aceptado:23 de octubre de 2019

DOI: http://dx.doi.org/10.21017/rimci.2020.v7.n13.a73

\begin{abstract}
Resumen
Velasco Ordóñez S.A.S. es una empresa dedicada a asesorías jurídicas, con lo cual su principal fuerza laboral está conformada por abogados, especializándose en Propiedad Intelectual y Derecho de Mercados. Con el fin de hacer una mejor utilización del tiempo de los mismos para así poder brindar una atención oportuna a todos los requerimientos recibidos por partes de los clientes, se desarrolló el proyecto con base en los lineamientos de la metodología BPM, consiguiendo finalmente, la presentación de tres posibles escenarios cuyas simulaciones y estructuraciones arrojaron nuevas posibles disponibilidades hasta de un 76\%, beneficiando así los tiempos de respuesta a los clientes y los niveles de productividad de la compañía.
\end{abstract}

Palabras clave. BPMS, Gestión de Procesos de Negocio; proceso; proceso crítico; reproceso; sobreproceso.

\begin{abstract}
Velasco Ordóñez S.A.S. is a company dedicated to legal advice, with which its main workforce is made up of lawyers, specializing in Intellectual Property and Market Law. In order to make better use of their time in order to provide timely attention to all the requirements received by customer parties, the project was developed based on the guidelines of the BPM methodology, finally getting the presentation of three possible scenarios whose simulations and structuring yielded new possible availabilities up to $76 \%$, thus benefiting customer response times and company productivity levels.
\end{abstract}

Key words. BPMS, Business Process Management; process; critical process; reprocessing; overprocess.

1 El artículo procede del proyecto de grado en Ingeniería Industrial denominado Estructuración del proceso de recepción, asignación y evacuación de requerimientos de trámites jurídicos de los clientes de la empresa Velasco Ordóñez S.A.S. mediante la metodología Business Process Management (BPM), de la Universidad Libre, realizado entre diciembre de 2016 y septiembre de 2019.

2 Estudiante X semestre Ingeniería Industrial, Universidad Libre, Bogotá, Colombia. Correo electrónico: luisa-gamezc@unilibre.du.co

3 Ingeniero industrial Universidad Libre, Bogotá, Colombia, MBE Herriut Watt University, Edinburgo, Escocia, docente Universidad Libre, Bogotá, Colombia Correo electrónico: ever.fuente@unilibre.edu.co 


\section{INTRODUCCIÓN}

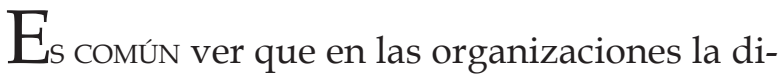
rección establece estrategias y objetivos encaminados a la satisfacción del cliente y, por otro lado, los diferentes departamentos de las mismas realizan sus procesos con unos distintos, es decir, buscan la realización de las funciones de cada uno procurando que su gestión sea eficiente, basados solo en el ser de cada una de las áreas, pero sin tener en cuenta que todos deben ir en una misma dirección. El éxito de una empresa radica en la posibilidad de trabajar como un todo organizado, como un Sistema en el que cada una de las partes integrantes trabajen en pro del bienestar general y no particular. BPM exige la alineación de esos objetivos, lo que aporta efectividad al negocio.

BPM tiene su centro en los procesos, ellos son el ser de esta metodología, y tienen una serie de componentes que es importante tener en cuenta y que se listan a continuación:

- Objetivo

- Alcance

- Entradas y salidas

- Elementos de entrada y salida

- Recursos

- Diagrama de Flujo

- Indicador de desempeño

La metodología BPM se desarrolla en determinadas etapas que conforman un ciclo, el cual se muestra en la Fig. 1.

Velasco Ordóñez S.A.S. es una empresa dedicada a la prestación de servicios de asesorías jurídicas. Precisamente, el tiempo es para Velasco Ordóñez S.A.S. su recurso más valioso y de su buen uso depende directamente el nivel de facturación mensual, teniendo dos situaciones: por un lado, entre más trabajos puedan realizarse mayor será lo facturado y, por otro, entre menos insatisfechos estén sus clientes menos probabilidad habrá de que piensen en retirarse y este punto es muy importante, puesto que el número de estos es reducido y la empresa actúa en un campo con mucha competencia.

La empresa tiene definidos diferentes procedimientos para realizar determinados procesos [2], como por ejemplo, apertura de casos, de clientes y

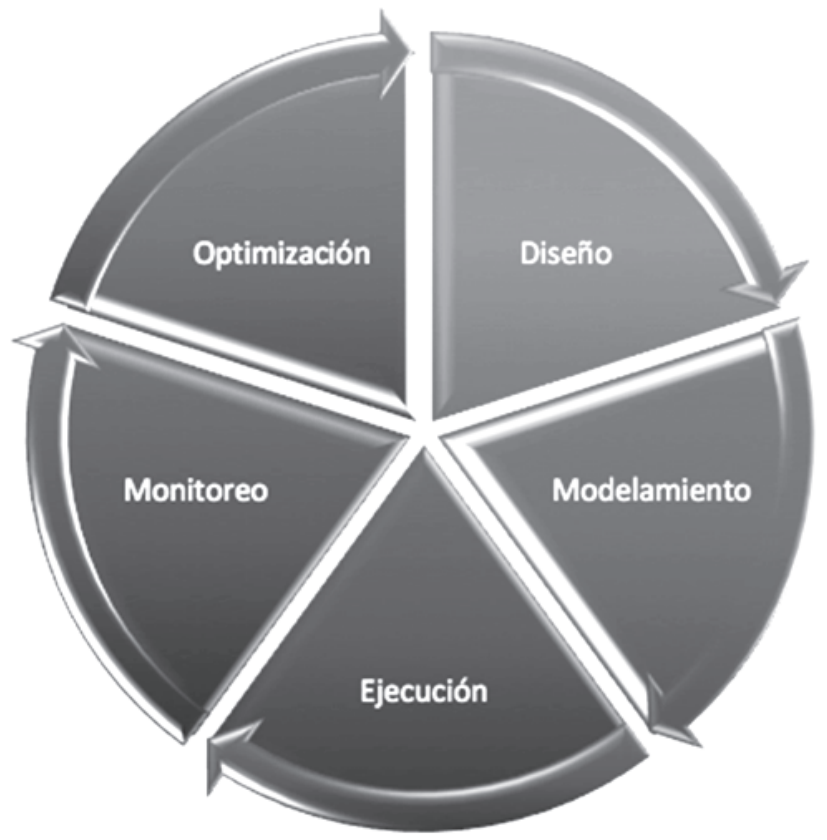

Fig. 1. Ciclo de vida BPM [1]. Fuente: Los autores, 2018. Basado en https://bpmsosw.wordpress.com/2012/02/12/ ciclo-de-vida-bpm/

carpetas físicas, diligencias de dependencia judicial, entre otros. En términos generales, siempre que llega un requerimiento el departamento de Coordinación de Información se encarga de recibir la solicitud, abrir el caso correspondiente en el sistema de gestión de expedientes, asignar la tarea a un abogado según los tiempos establecidos, este trabaja en el proyecto como corresponda, emite su concepto o trabajo final, lo envía a revisión del socio y, una vez aprobado, se envía el resultado al cliente.

No obstante, en algunas ocasiones la ejecución de esos procedimientos suele ser muy dispendiosa y no hay una interacción eficiente entre uno y otro, por lo que pueden ser demorados los tiempos totales de evacuación de las diferentes tareas asignadas, no solo en relación con los abogados sino también con el departamento de Coordinación de Información.

Actualmente, la empresa no lleva control de quejas y reclamos ni estadísticas sobre el cumplimiento de los requisitos del cliente. Las solicitudes de servicios llegan a través de correo electrónico y se llevan de esta manera todas las comunicaciones. Gracias al manejo escrito de los requerimientos recibidos se ha podido controlar el que se dé respuesta y trámite a cada uno de ellos.

Rev. Ingeniería, Matemáticas y Ciencias de la Información Vol. 7 / Núm. 13 / enero - junio de 2020; pág. 25-44 
Procurando implementar un sistema que permita el control de todas las requisiciones que se reciban, asegurando así que no se olviden o se demoren en ser contestadas, se estableció que todos los requerimientos de los clientes se dirigieran a un correo, el de la Coordinación de Información, cargo creado precisamente a raíz de la necesidad de centralizar las solicitudes recibidas y asignar las tareas a los abogados según la carga de trabajo de cada uno.

Hasta finales del año 2017, semanalmente se realizaba el "Comité de la Práctica", en el que se establecía el porcentaje de tareas cumplidas y vencidas de los abogados. Este comité daba una aproximación al nivel de satisfacción de los requerimientos de los clientes puesto que en él se totalizaba el número de tareas asignadas durante la semana a cada abogado y se determinaba el cumplimiento de cada uno según el número total de tareas efectivamente realizadas al día del comité.

Se estima que mientras haya un porcentaje de evacuación de tareas del $100 \%$ por parte de los abogados, los requerimientos de los clientes estarán siendo atendidos a satisfacción. Entre los meses de enero a marzo de 2017 el nivel de cumplimiento promedio del departamento jurídico es del $85 \%$ ) y seguían llegando por correo electrónico quejas de los clientes o solicitudes de agilidad en la tramitación de los requerimientos, a una razón promedio de 1 por semana. En la tabla I se muestra el comportamiento por comité de las tareas del equipo jurídico de los meses de enero a marzo de 2017.

Por políticas de la empresa se ha establecido que el tiempo de respuesta adecuado para los clientes es de máximo tres días hábiles y se trabaja en pro del cumplimiento de este término, pero cuando se excede o llegan requerimientos que deben ser resueltos de manera urgente se salta el orden de solución de las solicitudes recibidas, lo que provoca retrasos en la resolución de los trabajos recibidos y desorden en los procedimientos asociados a la evacuación de cada caso, incluido todo lo de valor agregado, incrementándose hasta en cinco o diez días más el tiempo total de evacuación de las tareas y generando caos en la realización de las actividades ordinarias. Esto deja en evidencia que no se cuenta con un plan de emergencia para la
Tabla I. Relación de tareas del equipo jurídico por comité

\begin{tabular}{|c|c|c|c|c|}
\hline $\begin{array}{c}\text { Comité } \\
\text { No. }\end{array}$ & $\begin{array}{c}\text { Total } \\
\text { tareas } \\
\text { asignadas }\end{array}$ & $\begin{array}{c}\text { Total } \\
\text { tareas } \\
\text { cumplidas }\end{array}$ & $\begin{array}{c}\text { Total } \\
\text { tareas } \\
\text { vencidas }\end{array}$ & $\begin{array}{c}\text { Porcentaje de } \\
\text { cumplimiento }\end{array}$ \\
\hline 141 & 322 & 267 & 55 & $83 \%$ \\
\hline 142 & 305 & 284 & 21 & $93 \%$ \\
\hline 143 & 315 & 296 & 19 & $94 \%$ \\
\hline 144 & 302 & 269 & 33 & $89 \%$ \\
\hline 145 & 316 & 243 & 73 & $77 \%$ \\
\hline 146 & 327 & 281 & 46 & $86 \%$ \\
\hline 147 & 396 & 384 & 12 & $97 \%$ \\
\hline 148 & 336 & 265 & 71 & $79 \%$ \\
\hline 149 & 349 & 269 & 80 & $77 \%$ \\
\hline 150 & 380 & 296 & 84 & $78 \%$ \\
\hline Promedio & 335 & 285 & 49 & $85 \%$ \\
\hline
\end{tabular}

Fuente: Los autores, basada en la información de Velasco Ordóñez S.A.S., 2017

recepción y trámite de "casos especiales" de inmediata atención (que suelen llegar con frecuencia).

La preocupación actual de la empresa reside en la satisfacción del cliente y en si se cuenta con los recursos necesarios, tanto físicos como humanos, para llevar a cabo las labores requeridas en el desarrollo de la actividad de la misma, o si se tiene un desperdicio de dichos recursos y del tiempo con el que cuentan para evacuar las solicitudes que reciben a diario. Esto, por cuanto se han establecido diferentes procedimientos, se han contratado distintos números de abogados (al mismo tiempo), se actualizan las funcionalidades del sistema de gestión de expedientes y se han creado nuevos cargos operativos que ayuden en la eficiencia del departamento jurídico (que es en el que se están presentando los inconvenientes de utilización del tiempo), como lo son el de Dependiente Judicial y el de Paralegal, y no por esto se han dejado de recibir quejas de los clientes ni de presentar retrasos en la entrega del trabajo final, con lo cual es importante para la organización determinar si hay maneras de obtener mejoras internamente con los recursos con que cuentan actualmente.

\section{MetodologíA}

El desarrollo del proceso metodológico se presenta en la tabla II, en el cual se presentan las diferentes actividades que se plantean para cada objetivo específico. 
Tabla II, Cuadro metodológico.

\begin{tabular}{|c|c|}
\hline OBJETIVOS ESPECÍFICOS & METODOLOGÍA \\
\hline $\begin{array}{l}\text { Identificar los procesos críticos que inciden en los niveles } \\
\text { de satisfacción de los clientes de Velasco Ordóñez S.A.S. } \\
\text { para el establecimiento de un diagnóstico en relación con } \\
\text { el grado de conocimiento que tienen los miembros de los } \\
\text { departamentos de Coordinación de Información y } \\
\text { Jurídico sobre los procedimientos generales relacionados } \\
\text { con el desarrollo y solución de los diferentes } \\
\text { requerimientos y la adecuada aplicación de los mismos }\end{array}$ & $\begin{array}{l}\text { A partir de entrevistas a los ejecutores de los procesos y de } \\
\text { una revisión documental de los procedimientos de la } \\
\text { empresa, se recabó información en relación con dichos } \\
\text { procesos y se elaboraron los diagramas correspondientes } \\
\text { [3]. Asimismo, se elaboró el diagnóstico de la situación } \\
\text { actual, a partir del uso de cuatro metodologías } \\
\text { establecidas para tal fin }\end{array}$ \\
\hline $\begin{array}{l}\text { Analizar la estructura de los diferentes procedimientos } \\
\text { documentados en la empresa y la interrelación entre } \\
\text { ellos, para la determinación de la pertinencia de los } \\
\text { mismos en relación con la respuesta a los requerimientos } \\
\text { y necesidades de los clientes. }\end{array}$ & $\begin{array}{l}\text { Se elaboró el cuadro de procesos de la empresa y el } \\
\text { diagrama de Ishikawa, luego de lo cual se procedió a su } \\
\text { análisis de los mismos en relación con su funcionamiento, } \\
\text { interacción y posibilidades de mejora }\end{array}$ \\
\hline $\begin{array}{l}\text { Estructurar mediante la aplicación de la metodología } \\
\text { Business Process Management (BPM) [4], procesos que le } \\
\text { permitan a Velasco Ordóñez S.A.S. el diseño, } \\
\text { modelamiento, organización, documentación y la } \\
\text { optimización continua de sus procesos, aumentando la } \\
\text { eficiencia de sus gestiones de valor agregado y del } \\
\text { resultado final al cliente, a través de una máxima } \\
\text { reducción de los tiempos de respuesta. }\end{array}$ & $\begin{array}{l}\text { Se analizaron los diferentes resultados obtenidos con } \\
\text { anterioridad con el fin de reestructurar algunos procesos } \\
\text { de manera tal que permitan a Velasco Ordóñez S.A.S. } \\
\text { aumentar la eficiencia de sus gestiones de valor agregado } \\
\text { y mejorar los tiempos de entrega del resultado final al } \\
\text { cliente, por lo cual se determinaron las variables a partir } \\
\text { de las cuales se trabajaría en la creación de los escenarios } \\
\text { de prospectiva. Luego de analizar la influencia de dichas } \\
\text { variables, se determinaron los tres posibles escenarios }\end{array}$ \\
\hline $\begin{array}{l}\text { Validar la pertinencia de los nuevos procesos } \\
\text { estructurados mediante la metodología BPM contra los } \\
\text { que se siguen actualmente en Velasco Ordóñez S.A.S. }\end{array}$ & $\begin{array}{l}\text { Se elaboraron los nuevos diagramas de flujo para ilustrar } \\
\text { la trayectoria que seguirían los requerimientos dentro de } \\
\text { la firma para poder ser tramitados y evacuados y se } \\
\text { hicieron las simulaciones correspondientes con el } \\
\text { programa Bizagi, tanto de los procesos actuales como de } \\
\text { los propuestos para los escenarios } 1 \text { y 2, para poder así } \\
\text { comparar a partir de los resultados obtenidos y } \\
\text { determinar un estimado de liberación de tiempo de los } \\
\text { abogados (metodología AS IS / TO BE) }\end{array}$ \\
\hline $\begin{array}{l}\text { Realizar la evaluación económica de los nuevos procesos } \\
\text { estructurados, determinando la viabilidad de su } \\
\text { implementación en Velasco Ordóñez S.A.S. }\end{array}$ & $\begin{array}{l}\text { Se realizó un flujo de caja para cada uno de los escenarios } \\
\text { propuestos y de la situación actual y expusieron los } \\
\text { comentarios correspondientes para que la empresa pueda } \\
\text { tomar una decisión }\end{array}$ \\
\hline
\end{tabular}

Fuente: Los autores, 2019

\section{DiágNOSTICO DE LA SITUACIÓN ACTUAL}

A partir del uso de cuatro metodologías, se obtuvo el diagnóstico de la situación actual. Estas cuatro metodologías son:

- Elaboración de la matriz Véster

- Análisis de las fuerzas competitivas de la empresa

- Análisis de los factores críticos de éxito, entendidos como aquellos que inciden directamente en el éxito o fracaso del negocio y que pueden ser controlados por la empresa
- Análisis DOFA (Debilidades, Oportunidades, Fortalezas y Amenazas) de la empresa

Con base en los resultados obtenidos de los cuatro diagnósticos elaborados, se tiene que, a raíz de los análisis DOFA[5] y de variables críticas de la empresa, se evidencia que esta debe prestar especial atención a los puntos relacionados a continuación:

- Retrasos o demoras

- Procedimientos establecidos

- Uso del tiempo

- Sistema de gestión de expedientes (SGA)

Rev. Ingeniería, Matemáticas y Ciencias de la Información Vol. 7 / Núm. 13 / enero - junio de 2020; pág. 25-44 
Lo anterior, debido a que, luego de hacer los análisis anteriormente indicados, se detectó que los inconvenientes presentados en la empresa tienen como punto central esos factores, así:

Se recibe un requerimiento el cual es evacuado a partir de unos procedimientos establecidos (que incluyen la completa actualización del SGA) dentro de un tiempo límite. Si se llega a presentar algo de último momento que altere el orden normal de las cosas se afectará necesariamente el tiempo inicial de respuesta al cliente, generando retrasos que conllevan a quejas inevitables de los mismos.

Por otro lado, la disponibilidad de tiempo también influye en la posibilidad de capacitación que requieren los miembros de la empresa para conocer los diferentes procedimientos y seguirlos adecuadamente [6]. En pro de cumplir con los tiempos establecidos, alcanzar los niveles de cumplimiento esperados y seguir los mencionados procedimientos, se deberá ejecutar el trabajo asignado con la mayor eficiencia posible para evitar reprocesos e incumplimientos con los clientes.

Ahora bien, a partir de los resultados obtenidos con el análisis de las fuerzas competitivas y los factores críticos de éxito se encuentra que los clientes son el punto crítico en el que debe centrarse la empresa en estos momentos. Precios competitivos y tiempos adecuados de respuesta son las variables a considerar, los cuales giran en torno a una utilización eficiente del tiempo hábil para laborar y ofrecer a la empresa una ventaja competitiva. Así, la clave podría ser el buen uso del tiempo con procesos pertinentes por parte del área jurídica quien, para el caso en particular, viene a ser la parte operativa de la firma.

Velasco Ordóñez S.A.S. es una empresa prestadora de servicios de asesoría jurídica, organizada en tres departamentos: Jurídico, Administrativo y de Información. Cada uno de los tres departamentos tiene funciones claramente establecidas y delimitadas y, si bien la metodología BPM enfoca el negocio hacia los procesos, se aclara que para el desarrollo del proyecto solo se tuvieron en cuenta aquellos relacionados con los departamentos Jurídico y de Información, puesto que, a partir de la elaboración del diagnóstico de la situación actual, se determina que estos son los que están incidiendo directamente en los resultados presentados a los clientes.

\section{AnÁlisis de pROCESOS DE LA SITUACIÓN ACTUAL}

Con el fin de obtener una vista general del esquema actual de la firma, se observaron las interacciones entre los diferentes procesos y departamentos para luego realizar la agrupación de los mismos.

Así las cosas, se determinó la relación de los tipos de procesos encontrados en la empresa y se construyó el mapa de procesos de Velasco Ordóñez S.A.S., según se muestra a continuación, en la Fig. 2.

Asimismo y con el fin de poder observar la situación actual desde otro punto de vista, se realizó el diagrama de Ishikawa a partir de la información obtenida en las encuestas y de lo observado al trabajar con los procedimientos y procesos, el cual se muestra a continuación, en la Fig. 3.

Finalmente, se elaboró un manual de procedimientos y se procedió al análisis de los procesos, su funcionamiento, interacción y posibilidades de mejora. Al respecto, se pudieron establecer los puntos indicados a continuación:

- A partir del cuadro de procesos y el manual de procedimientos se detectó que, si bien los procesos misionales son en los que se centra el presente proyecto, es importante tener en cuenta los relacionados con la Gerencia estratégica, puesto que su participación en el desarrollo de los primeros puede afectar el resultado del trabajo de los mismos.

- Hay varios procedimientos que se pueden agrupar para reducir el número de procesos llevados a cabo en la realización de diferentes actividades, lo cual podría beneficiar a la reducción de los tiempos de espera del cliente.

- A partir de la elaboración del Diagrama de Ishikawa, se observa que hay posibilidades de mejora a través de la capacitación del personal, tanto en el manejo de las herramien- 


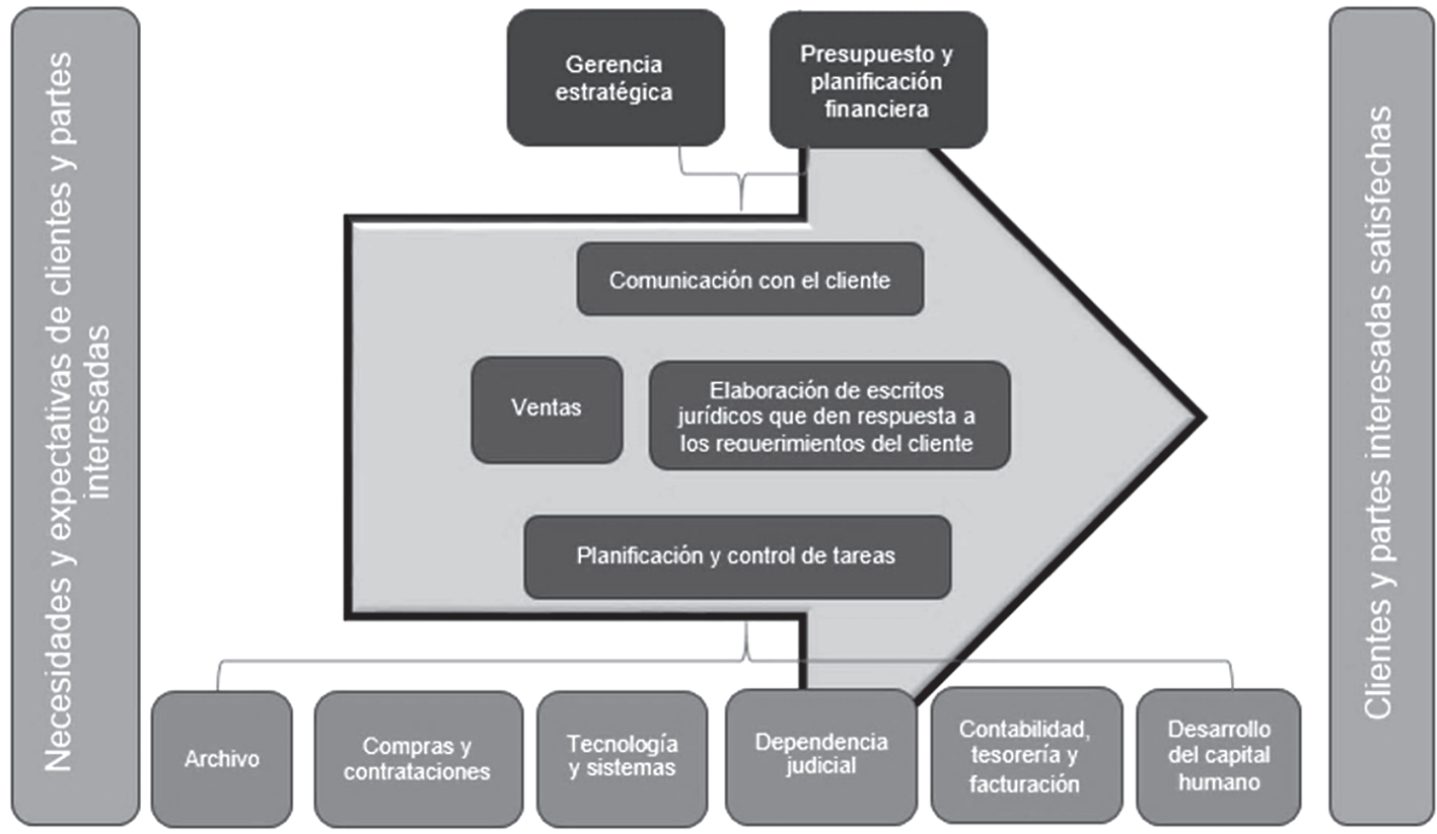

Fig.2. Mapa de procesos de Velasco Ordóñez S.A.S. Fuente: Los autores, basada en la información de Velasco Ordóñez S.A.S., 2019

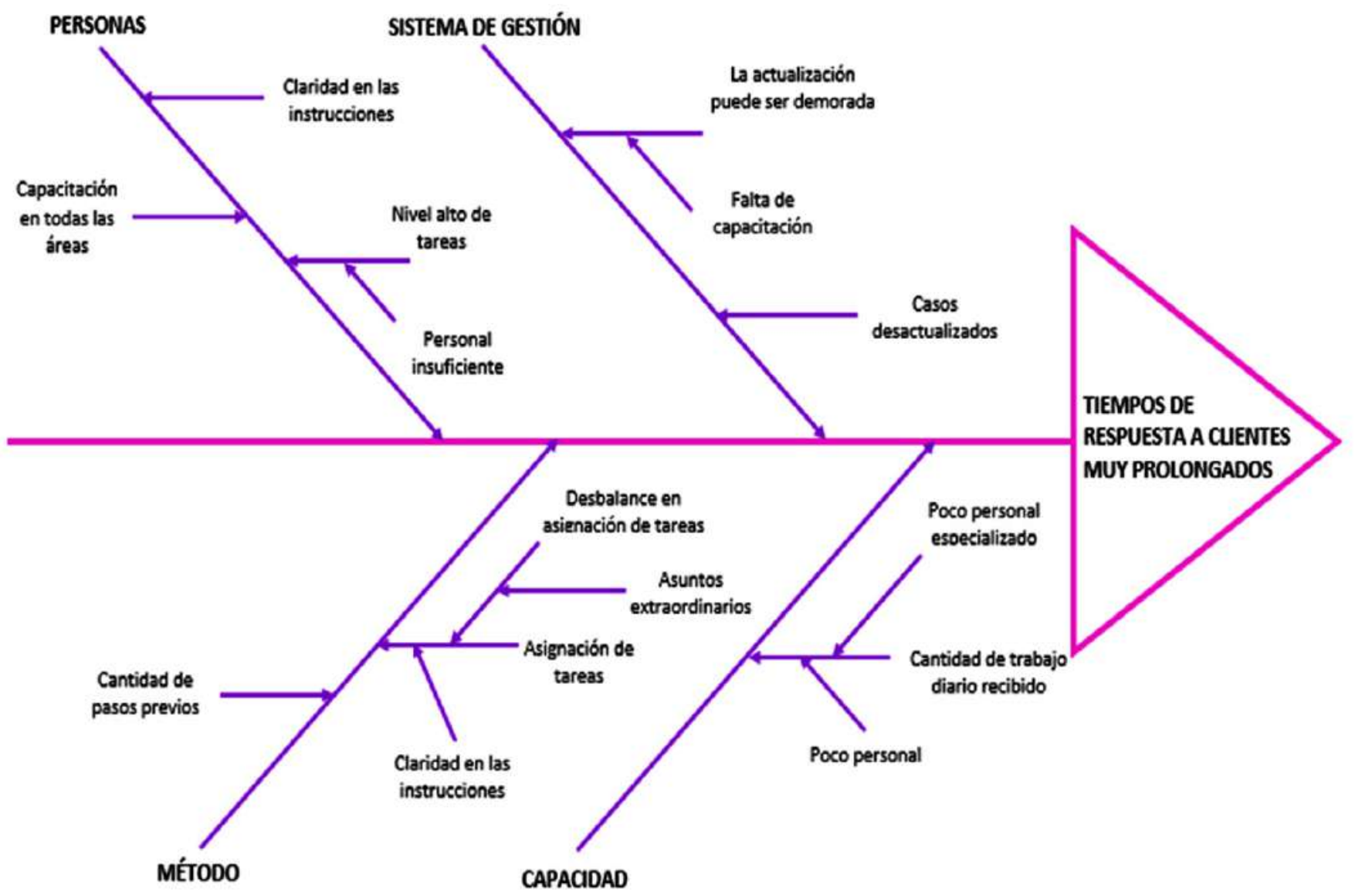

Fig. 3. Diagrama de Ishikawa de Velasco Ordóñez S.A.S. Fuente: Los autores, basada en la información de Velasco Ordóñez S.A.S., 2019 
tas como en las competencias asociadas a sus cargos, y en la asignación de tareas.

- Es importante determinar, desde los perfiles de cargos, la necesidad o no de la especialización de los abogados para, de esta manera, poder realizar una asignación balanceada de tareas. En este punto es preciso aclarar que el balance no está única y/o exclusivamente relacionado con la cantidad de tareas asignadas, sino con lo que su ejecución representa en tiempo y recursos para la empresa. De esta manera, la especialización de los abogados puede permitir que el que sea experto en un tema lo trabaje y pueda entregar, de manera rápida y acertada, un resultado esperado. Por otro lado, la no especialización puede brindar la posibilidad de que los encargos puedan ser realizados por cualquier miembro del equipo jurídico, sin limitar su ejecución a la dependencia o disponibilidad de una persona en particular.

- Luego de determinar lo anterior, es importante que las tareas sean asignadas siguiendo unos parámetros establecidos con anterioridad, facilitando así una ejecución adecuada de las tareas.

- Se requiere la concentración del trabajo especializado en manos del equipo jurídico, aunque no necesariamente en los profesionales. Aquello que no requiera atención especial de un abogado puede ser asignado a otro miembro del equipo que pueda dedicarse a esas labores y libere tiempo para la ejecución y entrega final de resultados al cliente.

- Es importante la implementación de formatos y parámetros a lo largo de todo el proceso, que permitan la unificación de criterios a tener en cuenta en la ejecución de las diferentes actividades, facilitando la comunicación a lo largo de los procesos. De esta manera se podría llegar a disminuir, bien sea el número de elementos que requieran revisión del Socio o bien el número de trabajos a corregir, así como los reprocesos a lo largo de toda la cadena.

\section{ESCENARIOS PROPUESTOS}

Luego de estudiar la situación actual de la empresa, se analizaron los diferentes resultados obtenidos con el fin de reestructurar algunos procesos de manera tal que permitan a Velasco Ordóñez S.A.S. aumentar la eficiencia de sus gestiones de valor agregado y mejorar los tiempos de entrega del resultado final al cliente. De acuerdo con esto, se determinaron las variables a partir de las cuales se trabajará en la creación de los escenarios de prospectiva.

Ahora bien, con el fin de determinar el nivel de influencia de estas variables, se calificaron según su impacto bajo los criterios de: Posicionamiento en el mercado, calidad del servicio prestado, precios de los servicios, costos de producción (dado en términos del tiempo invertido) y niveles de productividad de la empresa.

En la tabla III se muestran las variables que influyen en la situación actual:

En relación con el impacto sobre los niveles de productividad de la empresa, las cinco variables tecnológicas son calificadas como muy influyentes, en tanto que para las no tecnológicas el tiempo final de respuesta al cliente se encontró poco influyente. Esto se explica porque, de requerirse respuestas inmediatas, se organizará el trabajo de manera tal en que se logre satisfacer al cliente; es decir, los trabajos finales serán entregados, aunque el desarrollo de las actividades internas se altere.

En cuanto al impacto sobre la calidad del servicio prestado, las variables, tanto tecnológicas como no tecnológicas, son consideradas como influyentes, a excepción de la capacitación en el manejo del sistema de gestión de expedientes SGA. Esto se entiende porque, al ser un trabajo realizado en equipo, las falencias en el manejo del sistema por parte de algún empleado son suplidas por las habilidades de algún otro. Si bien esto no afecta directamente al cliente, sí puede llegar a generar inconvenientes en el desarrollo de los procedimientos.

Así, en la Fig. 4 se muestra el resultado obtenido para las variables tecnológicas y en la Fig. 5 el 
Tabla III. Variables con incidencia directa en la situación actual de Velasco Ordóñez S.A.S.

\begin{tabular}{|c|c|c|}
\hline VARIABLE & DEFINICIÓN & ESTADO ACTUAL \\
\hline $\begin{array}{l}\text { Estructuración de } \\
\text { procesos que organicen } \\
\text { los procedimientos } \\
\text { establecidos }\end{array}$ & $\begin{array}{l}\text { Hace referencia a la estandarización de } \\
\text { procesos, establecimiento de formatos y pasos } \\
\text { que permitan realizar el trabajo bajo criterios } \\
\text { unificados, disminuyendo los reprocesos }\end{array}$ & $\begin{array}{l}\text { Si bien se cuenta con unos parámetros } \\
\text { establecidos, en muchos casos se requiere } \\
\text { de la opinión de uno o más miembros del } \\
\text { equipo para avanzar en la ejecución de las } \\
\text { tareas, generando retrasos y } \\
\text { desinformación }\end{array}$ \\
\hline $\begin{array}{l}\text { Disposición de la } \\
\text { información de los } \\
\text { casos }\end{array}$ & $\begin{array}{l}\text { Corresponde al almacenamiento de la } \\
\text { información, bien sea en medio físico y/o } \\
\text { digital, de manera tal que todos los } \\
\text { intervinientes puedan acceder a la misma }\end{array}$ & $\begin{array}{l}\text { De preferencia se almacena la mayor } \\
\text { cantidad de información posible por caso } \\
\text { en el SGA, pero esto depende de que cada } \\
\text { miembro del equipo actualice } \\
\text { correspondientemente el caso en el } \\
\text { momento en que lo está trabajando }\end{array}$ \\
\hline $\begin{array}{l}\text { Actualización de los } \\
\text { sistemas informativos }\end{array}$ & $\begin{array}{l}\text { Establece los parámetros bajo los cuales se debe } \\
\text { mantener actualizado el SGA y el archivo físico }\end{array}$ & $\begin{array}{l}\text { Está más actualizado el SGA que el } \\
\text { archivo físico. En algunos casos no todos } \\
\text { los documentos del archivo físico han sido } \\
\text { incorporados en el SGA }\end{array}$ \\
\hline $\begin{array}{l}\text { Capacitación en el } \\
\text { manejo del sistema de } \\
\text { gestión de expedientes } \\
\text { - SGA }\end{array}$ & $\begin{array}{l}\text { Se refiere al estado de capacitación con el que } \\
\text { cuentan los diferentes miembros del equipo y el } \\
\text { nivel de conocimiento que tienen sobre el uso } \\
\text { del SGA }\end{array}$ & $\begin{array}{l}\text { Las capacitaciones deben ser frecuentes } \\
\text { puesto que se ofrecen solo en el momento } \\
\text { en que surgen dudas sobre determinados } \\
\text { asuntos. No hay jornadas de capacitación } \\
\text { establecidas }\end{array}$ \\
\hline Asignación de tareas & $\begin{array}{l}\text { Comprende los criterios a tener en cuenta para } \\
\text { la asignación de diferentes actividades a los } \\
\text { distintos miembros del equipo }\end{array}$ & $\begin{array}{l}\text { Se asignan tareas preferiblemente según el } \\
\text { conocimiento y la experticia de cada } \\
\text { miembro, pero esto genera sobrecargas en } \\
\text { algunos casos puesto que hay épocas en } \\
\text { las que unos temas se mueven más que } \\
\text { otros }\end{array}$ \\
\hline $\begin{array}{l}\text { Disponibilidad para la } \\
\text { atención de casos } \\
\text { especiales }\end{array}$ & $\begin{array}{l}\text { Hace referencia a los procedimientos dispuestos } \\
\text { para atender casos de atención extraordinaria, } \\
\text { sin alterar el funcionamiento normal y la } \\
\text { ejecución de los trabajos adicionales }\end{array}$ & $\begin{array}{l}\text { No se cuenta con un procedimiento de } \\
\text { emergencia para atender estos casos, se } \\
\text { asignan según disponibilidad y } \\
\text { conocimiento del tema }\end{array}$ \\
\hline $\begin{array}{l}\text { Tiempos } \\
\text { implementados en la } \\
\text { realización de las } \\
\text { actividades }\end{array}$ & $\begin{array}{l}\text { Se refiere al tiempo invertido por los miembros } \\
\text { del equipo en diferentes tareas que permitan } \\
\text { dar respuesta a los requerimientos de los } \\
\text { clientes }\end{array}$ & $\begin{array}{l}\text { Se requiere una redistribución de las } \\
\text { actividades para optimizar los tiempos } \\
\text { implementados en cada una de ellas por } \\
\text { los diferentes miembros del equipo }\end{array}$ \\
\hline $\begin{array}{l}\text { Tiempos de respuesta } \\
\text { a los clientes }\end{array}$ & $\begin{array}{l}\text { Se refiere al tiempo que transcurre desde el } \\
\text { momento en que se recibe un requerimiento } \\
\text { hasta cuando se le ha dado la correspondiente } \\
\text { respuesta al cliente }\end{array}$ & $\begin{array}{l}\text { Las demoras en la realización de las } \\
\text { diferentes actividades genera un } \\
\text { consecuente retraso en la respuesta final al } \\
\text { cliente }\end{array}$ \\
\hline
\end{tabular}

Fuente: Los autores, 2019 


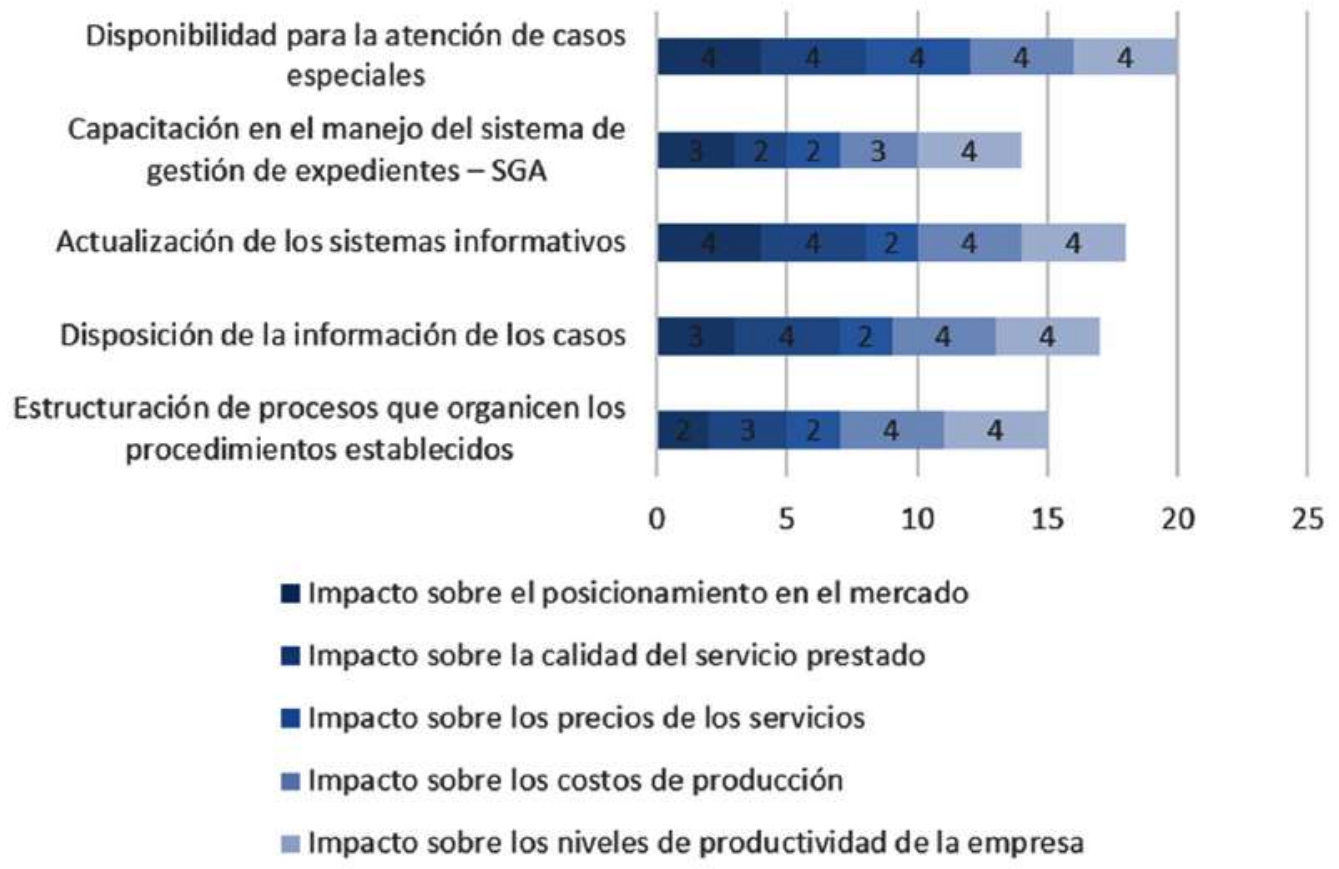

Fig 4. Impacto de las variables tecnológicas. Fuente: Los autores, basada en la información de Velasco Ordóñez S.A.S., 2019

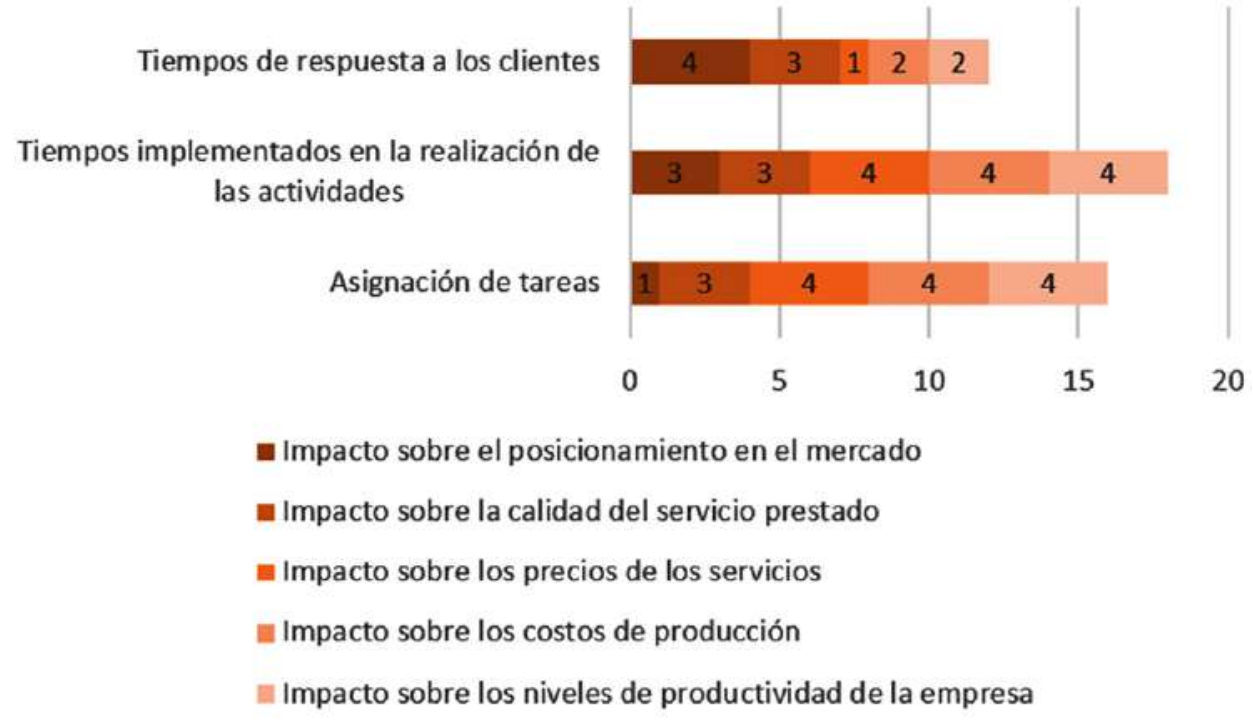

Fig. 5. Impacto de las variables no tecnológicas. Fuente: Los autores, basada en la información de Velasco Ordóñez S.A.S., 2019

de las variables no tecnológicas, teniendo como 4 el mayor nivel de influencia y como 1 el menor, con 2 y 3 como los intermedios correspondientes.

A partir de lo anterior, se realizó un árbol de problemas a modo de resumen de la situación actual, el cual se muestra en la Fig. 6.
Con base en lo anterior, se determinó que los procesos a sugerir podrían estructurarse a partir de las siguientes prioridades identificadas:

- Concentrar el trabajo especializado en manos del equipo jurídico, aunque no necesariamente en los profesionales. 


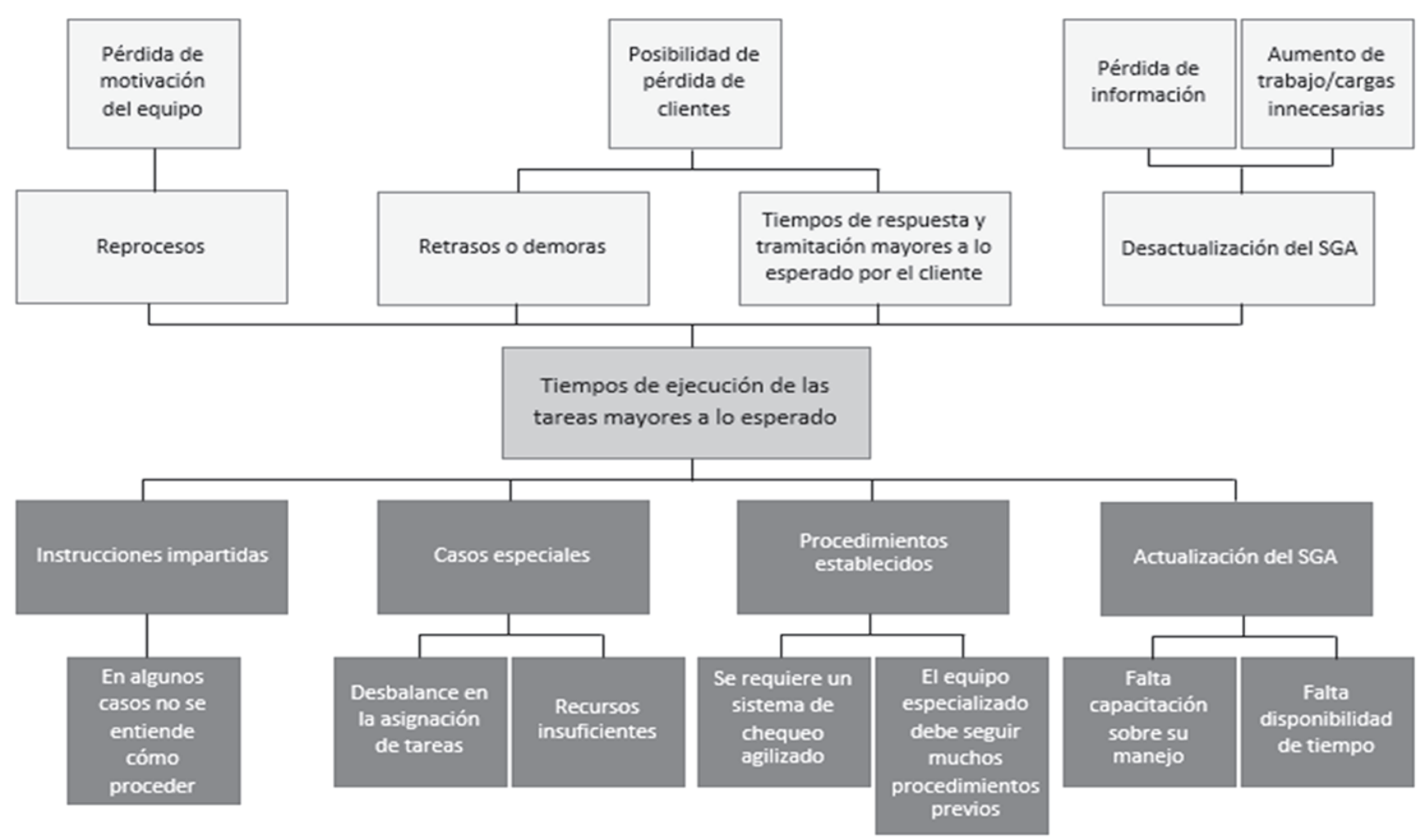

Fig. 6 Arbol de problemas de la situación actual de Velasco Ordóñez S.A.S. Fuente: Los autores, basada en la información de Velasco Ordóñez S.A.S., 2019

- Implementar formatos y parámetros a lo largo de todo el proceso.

- Realizar una asignación balanceada de tareas, teniendo en cuenta la disponibilidad de los diferentes tipos de recursos, incluyendo las capacidades de mano de obra y experticia.

Ahora bien, revisando los antecedentes y el árbol elaborado, se evidencia que el problema central es el tiempo y la inversión del mismo. De acuerdo con esto, durante el mes de mayo de 2018 se elaboró un informe de tiempos invertidos del equipo jurídico durante los meses de enero y abril del mismo año. A continuación, se indican las conclusiones más relevantes que serán tenidas en cuenta para la reestructuración sugerida:

- Del total del tiempo facturado por los abogados, el 38,44\% (580,13 horas) se invierte en trabajos directamente facturables; el $61,56 \%$ (929,08 horas) corresponde a trabajos que no se traducirán en una inmediata factura al cliente.
- De los trabajos que no se traducen directamente en una factura al cliente, es decir, lo "no facturable", los diez primeros que más dedicación de tiempo requieren son:

1. Elaboración de reportes

2. Avance de trámite o solicitud de impulso para dar continuidad a los trámites

3. Investigaciones / revisión de antecedentes

4. Revisión de gaceta

5. Envío de correo a cliente

6. Actualización de SGA

7. Reuniones del equipo

8. Comité diario

9. Consultas internas

10.Elaboración de avisos de posible oposición

Teniendo en cuenta toda la información obtenida y con el fin de poder establecer finalmente tres posibles escenarios, en aras de disminuir el tiempo invertido por los abogados en la realización del trabajo especializado, se realizó un análisis IGO (importancia y gobernabilidad), en el que se asignó una calificación a cada una de las ocho

Rev. Ingeniería, Matemáticas y Ciencias de la Información Vol. 7 / Núm. 13 / enero - junio de 2020; pág. 25-44 
variables según: Nivel de importancia, de 1 a 4, y de gobernabilidad, de 1 a 5, tal como se muestra en la tabla IV.

Los resultados del análisis fueron depositados en la matriz indicada en la Fig. 7.

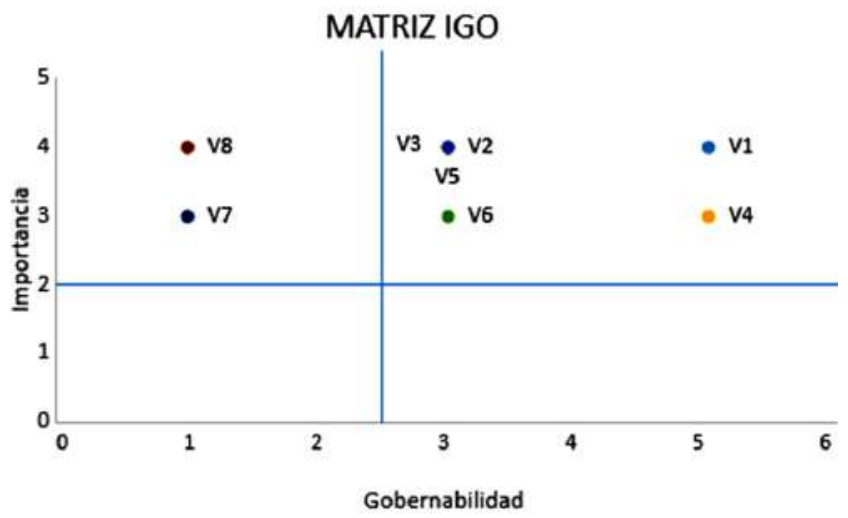

Fig. 7. Matriz IGO de las variables con incidencia directa [7][8] en la situación actual de Velasco Ordóñez S.A.S. Fuente: Los autores, basada en la información de Velasco Ordóñez S.A.S., 2019

Las variables localizadas en el extremo superior derecho son consideradas como las que requieren atención inmediata y las ubicadas en el extremo superior izquierdo representan un reto puesto que, si bien repercuten en el problema, no se tiene un control total sobre las mismas al depender del tiempo implementado finalmente por cada miembro del equipo, el cual a su vez depende de su nivel de experticia, concentración y otras características más que afectan a cada empleado en el desarrollo de sus funciones [9].

Como lo que se quiere es precisamente ayudar a que esos tiempos empleados por cada miembro del equipo puedan ser mejorados, la empresa sí puede brindar herramientas que faciliten el desempeño de sus funciones, con base en las seis variables de atención inmediata, las cuales pasan a ser consideradas incertidumbres para fijar los escenarios prospectivos. Así, se realizó el análisis morfológico de las variables para determinar los escenarios a proponer, según se muestra en la Fig. 8.

En aras de disminuir el tiempo invertido por los abogados en la realización del trabajo especializado, en el análisis se establecen tres posibles escenarios.

Escenario 1: Plantear la distribución del trabajo a los abogados a partir de la especialización del mismo, es decir, asignación del trabajo según las competencias y habilidades particulares de cada abogado o según los diferentes temas abordados por la compañía.

Escenario 2: Reorganizar la estructura del equipo jurídico actual. Esto incluye el estudio y suge-

Tabla IV. Análisis IGO de las variables con incidencia directa en la situación actual de Velasco Ordóñez S.A.S.

\begin{tabular}{|c|c|c|c|c|c|}
\hline \multicolumn{6}{|c|}{ Resultados utilización de recursos } \\
\hline \multicolumn{2}{|c|}{ Situación actual } & \multicolumn{2}{|l|}{ Escenario 1} & \multicolumn{2}{|c|}{ Escenario 2} \\
\hline Recurso & Uso & Recurso & Uso & Recurso & Uso \\
\hline Socio & $41,85 \%$ & Socio & $13,81 \%$ & Socio & $9,67 \%$ \\
\hline $\begin{array}{l}\text { Coordinador de } \\
\text { Información }\end{array}$ & $11,41 \%$ & Abogado Nivel 3 & $39,50 \%$ & Abogado Nivel 3 & $23,98 \%$ \\
\hline $\begin{array}{l}\text { Analista de } \\
\text { Información }\end{array}$ & $1,82 \%$ & Abogado Nivel 2 Regulatorio y Procesal & $77,24 \%$ & $\begin{array}{l}\text { Coordinador de } \\
\text { Información }\end{array}$ & $1,08 \%$ \\
\hline \multirow[t]{6}{*}{ Abogados } & $98,54 \%$ & $\begin{array}{l}\text { Abogado Nivel } 2 \text { Propiedad Intelectual. Comercial } \\
\text { y Administrativo }\end{array}$ & $70,25 \%$ & Paralegales & $2,97 \%$ \\
\hline & & Abogado Nivel 1 Regulatorio y Procesal & $81,28 \%$ & Abogado Nivel 2 & $23,54 \%$ \\
\hline & & $\begin{array}{l}\text { Abogado Nivel } 1 \text { Propiedad Intelectual. Comercial } \\
\text { y Administrativo }\end{array}$ & $97,98 \%$ & Abogado Nivel 1 & $99,24 \%$ \\
\hline & & Paralegal Regulatorio y Procesal & $5,10 \%$ & & \\
\hline & & $\begin{array}{l}\text { Paralegal Propiedad Intelectual. Comercial y } \\
\text { Administrativo }\end{array}$ & $5,50 \%$ & & \\
\hline & & Coordinador de Información & $1,45 \%$ & & \\
\hline
\end{tabular}

Fuente: Los autores, 2019 


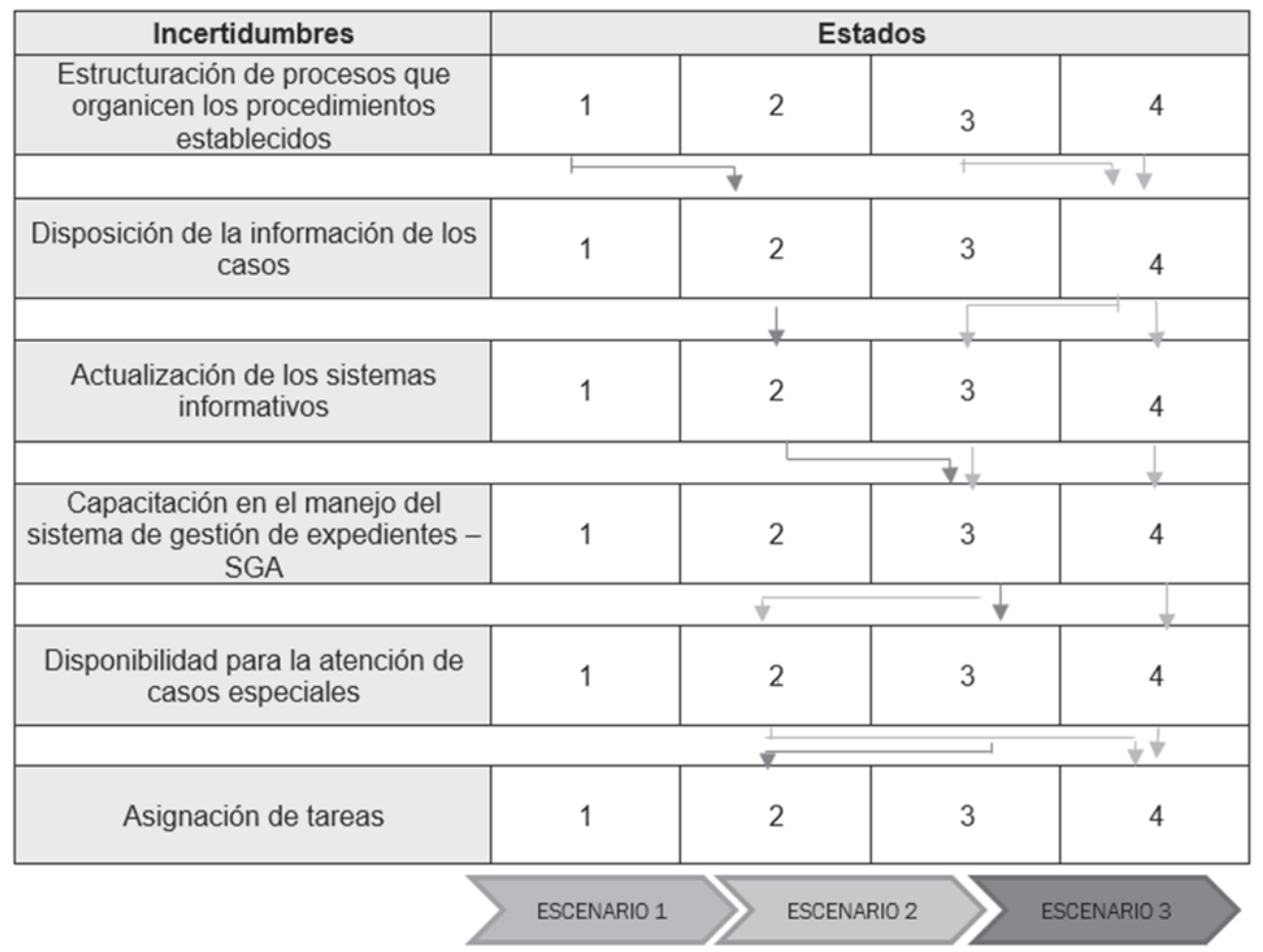

Fig. 8. Matriz de Análisis Morfológico. Fuente: Los autores, basada en la información de Velasco Ordóñez S.A.S., 2019

rencia de nuevos posibles cargos para redistribuir la realización de las diferentes actividades con el fin de dejar en manos de los abogados el trabajo que solo pueda y deba ser realizado por ellos, permitiendo la eficiencia en el uso de su tiempo.

Escenario 3: Reorganizar actividades de manera tal que se asignen algunas a otros miembros del equipo, permitiendo que los abogados se concentren en realizar aquello que solo ellos podrían ejecutar y que es el objeto en sí de los servicios prestados por la empresa. Al respecto, se hace la observación de que se puede terminar recargando uno o más miembros actuales del equipo, lo que generaría efectos secundarios que afecten de alguna manera la organización, como la desactualización del SGA.

Cabe anotar que en el escenario 2 se planteará la distribución del trabajo a los abogados según la carga de cada uno, balanceando la entrega de proyectos (repartición igualitaria). Por otro lado, en el escenario 1 se estudiará la asignación del trabajo según las destrezas, competencias y habilidades de cada abogado en los diferentes campos de la compañía.

\section{A. Diseño y presentación del escenario 1}

En la figura 9 se muestra el cargograma actual del departamento jurídico en Velasco Ordóñez S.A.S.

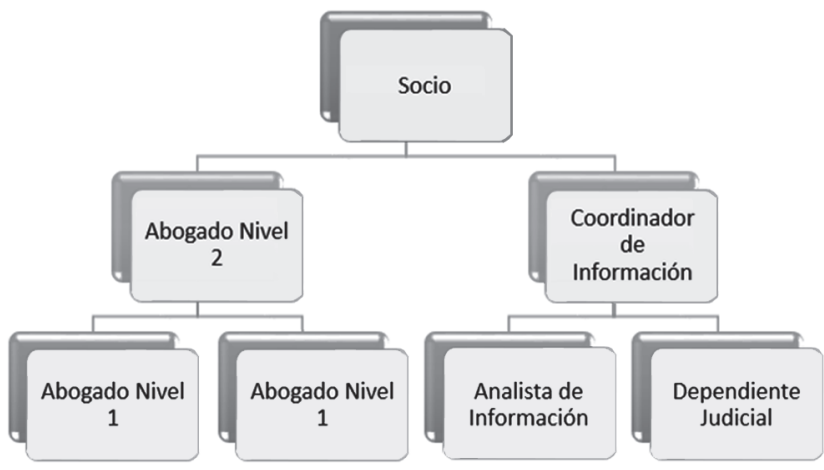

Fig. 9. Cargograma actual del departamento jurídico en Velasco Ordóñez S.A.S. Fuente: Los autores, basada en la información de Velasco Ordóñez S.A.S., 2019

Se propone establecer responsables de área o, dicho de otra manera, dividir las actividades de manera tal que el trabajo recibido sea asumido distintamente por un grupo, como se muestra en la Fig. 10.

Con esta nueva estructura se busca:

Rev. Ingeniería, Matemáticas y Ciencias de la Información Vol. 7 / Núm. 13 / enero - junio de 2020; pág. 25-44 


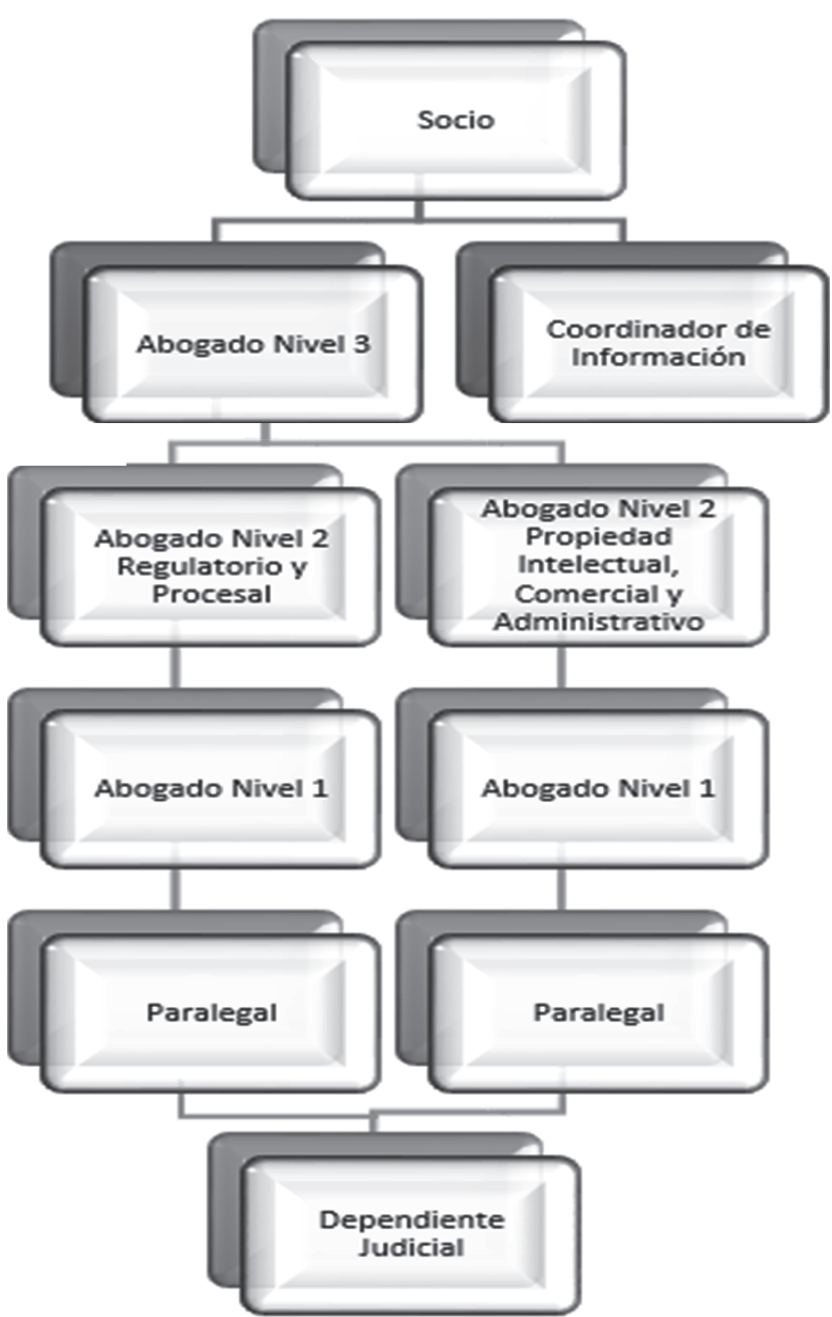

Fig. 10. Cargograma propuesto del departamento jurídico en Velasco Ordóñez S.A.S. por áreas de acción. Fuente: Los autores, basada en la información de Velasco Ordóñez S.A.S., 2019

- Especializar a los abogados para que la realización del trabajo sea mucho más rápida y fluida, al necesitar invertir menos tiempo en capacitación e investigación.

- Repartir el trabajo recibido de manera tal que pueda haber cargas equilibradas.

- Brindarle a los clientes resultados mucho más óptimos y con menos tiempos de respuesta. Por ejemplo, el abogado encargado de sanitario tendrá más disponibilidad de dar respuesta a consultas de los clientes si no debe pasar de una demanda de competencia desleal, a un proyecto de patentes para terminar prestando atención al requerimiento sanitario. Estar conectado en un mismo tema permitiría mayor fluidez y un menor desgaste.

\section{B. Diseño y presentación del escenario 2}

Con base en el informe de horas invertidas no solo en aquello no facturable sino también en lo facturable, se determina que es posible invertir mejor el tiempo de los abogados si se concentra en ellos el trabajo especializado, aquello que requiere de un mayor criterio jurídico.

En la Fig. 11 se muestra el cargograma sugerido para el departamento jurídico en Velasco Ordóñez S.A.S. Con esto se busca aumentar la capacidad operativa del equipo puesto que, analizando los tiempos facturados, se encuentra que hay una gran cantidad de actividades que deben realizarse para poder cumplir con los requerimientos

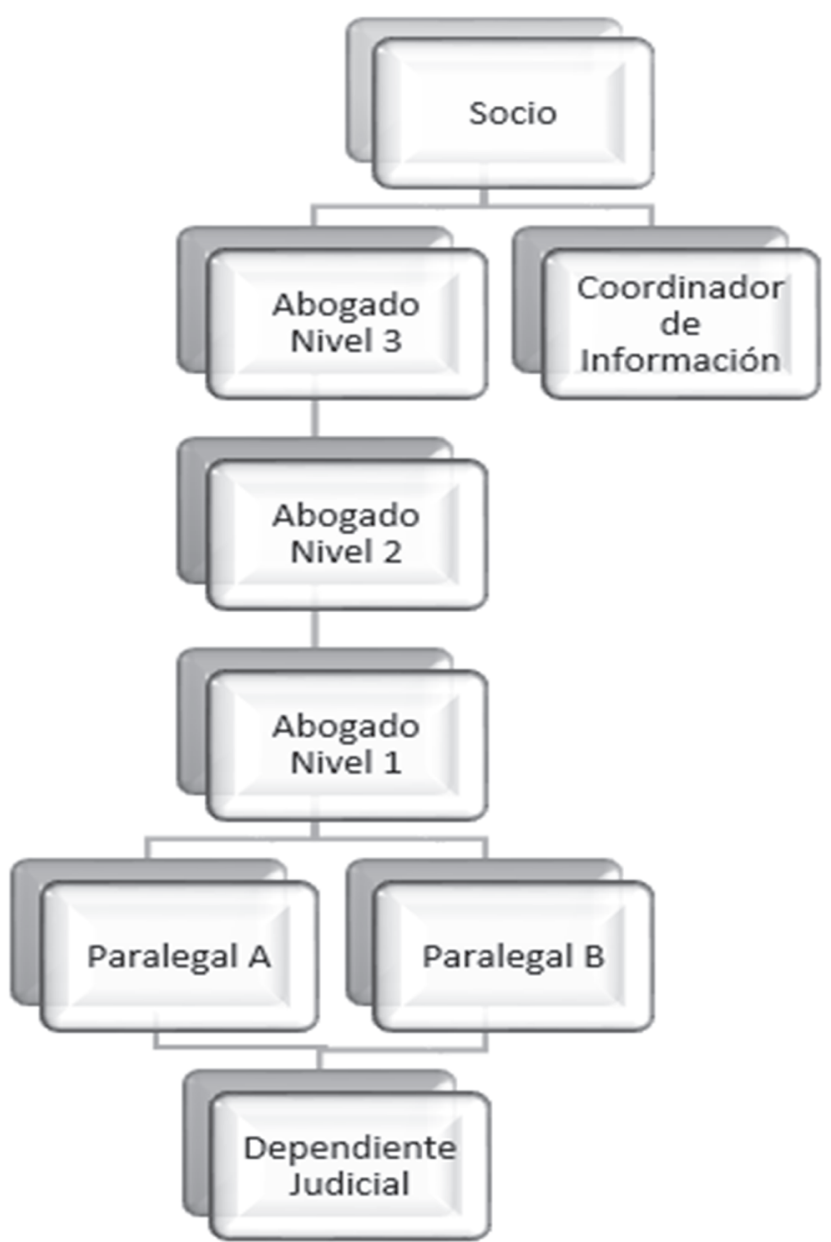

Fig. 11. Cargograma propuesto del departamento jurídico en Velasco Ordóñez S.A.S. Fuente: Los autores, basada en la información de Velasco Ordóñez S.A.S., 2019 
de los clientes y mantener actualizados los sistemas operativos, pero también hay distintos niveles de complejidad en aquellos trabajos que deben ser realizados por los abogados, por eso se plantea la posibilidad de reorganizar el departamento para reasignar algunas funciones y lograr una mayor efectividad en la consecución de los resultados esperados.

Así las cosas, no solo se plantea un refuerzo en la parte operativa del departamento sino una estratificación dentro del equipo especializado, para que se aproveche mejor el conocimiento y la experticia de los abogados, logrando una inversión de tiempo más productiva.

Con esta nueva estructura se busca:

- Reducir al máximo la cantidad de trabajos a revisar por parte del Socio, a la vez aumentar la calidad del mismo, puesto que se espera que la revisión del Abogado Nivel 3 genere aportes de fondo a los documentos.

- Concentrar el trabajo de manera tal que se aproveche al máximo la capacidad de cada abogado para ejecutar el trabajo facturable.

- Ampliar la parte operativa del departamento y mejorar su perfil, liberando tiempo a los abogados y logrando mayor agilidad en la realización de las actividades no facturables.

- Concentrar todos los temas jurídicos en el departamento para evitar fallos procedimentales de fondo, por esta razón se sugiere que el Dependiente Judicial trabaje directamente bajo las indicaciones de los paralegales.

Incluso se puede considerar la posibilidad de agregar otro Abogado Nivel 1 y repartir de manera equitativa las cargas entre los dos, aunque hasta este momento se considera que, dividiendo el trabajo según nivel de responsabilidades y especialización en el conocimiento de los temas, se puede lograr una repartición balanceada del trabajo actual recibido. No obstante, es bueno aclarar que, en caso de aumentar el nivel de trabajo, con esta estructura sería fácil determinar a futuro qué perfil se requiere según donde se comience a detectar un cuello de botella [10].

\section{Diseño y presentación del escenario 3}

Para el diseño de este escenario se toma como base el informe de tiempos, a partir del cual se revisan aquellas actividades que pueden dejar de ser realizadas por los abogados sin afectar el resultado final a entregar al cliente, por el contrario, se busca aumentar su disponibilidad para que los abogados puedan ejecutar aquello que requiere mayor atención para que su producto final sea el mejor posible.

Así las cosas, se extrae el listado de trabajos agrupados en facturables y no facturables, con el fin de poder realizar el análisis correspondiente. $\mathrm{Al}$ respecto, es importante tener en cuenta que al totalizar $100 \%$ se hace referencia al peso de cada ítem dentro de su clasificación correspondiente, es decir, el total dentro del 38,44\% del tiempo total invertido en asuntos facturables y el total dentro del $61,56 \%$ del tiempo total invertido en asuntos no facturables.

Lo que se busca en este escenario es concentrar la mayor cantidad posible de trabajo facturable en los abogados y reasignar el no facturable en los demás miembros del equipo, es decir, Analista de Información, Coordinador de Información y Dependiente Judicial.

Se indicó para cada actividad la sugerencia de quién puede realizarla en lugar de un abogado, lo que se requiere para que esto se pueda ejecutar sin presentar una inferencia negativa en el resultado final entregado al cliente y el porcentaje estimado que el abogado invertirá en el desarrollo de cada actividad.

De acuerdo con lo anterior, se estima una liberación del tiempo en un $32,33 \%$, con lo cual se aumenta la disponibilidad para realizar más trabajos facturables. No obstante, también es importante tener en cuenta que esto aumentaría la carga para las personas que reciben el trabajo reasignado, lo que podría llegar a afectar en un corto plazo a algunos procesos de soporte o apoyo, repercutiendo directamente en los procesos misionales.

Ahora bien, al analizar la información del escenario 3 se puede notar que:

Rev. Ingeniería, Matemáticas y Ciencias de la Información Vol. 7 / Núm. 13 / enero - junio de 2020; pág. 25-44 
- Es posible reducir el tiempo invertido en asuntos no facturables al reasignar actividades a otros miembros del equipo.

- La reasignación del trabajo a personas que ya tienen funciones definidas y en las cuales invierten el $100 \%$ de su tiempo, es decir, cuya capacidad de trabajo está al tope, generará un desbalance en la operación.

- Si bien se estima un aumento en el tiempo disponible para realizar trabajos facturables, $32,33 \%$, ese porcentaje no necesariamente garantiza que se dará una respuesta más rápida a los clientes puesto que, al considerar que se dispone de más tiempo, se recibirá mayor cantidad de trabajo, volviendo rápidamente a presentar tiempos de respuesta muy largos a los requerimientos de los clientes.

- Dentro del trabajo facturable se encuentran diferentes niveles de actividades, desde las más complejas hasta las menos complejas que, si bien requieren ser realizadas por un profesional en Derecho debido a la necesidad del criterio jurídico, se pueden reasignar según el nivel de complejidad.

\section{Simulación de los escenarios PROPUESTOS}

A partir de los escenarios propuestos, se elaboraron los nuevos diagramas de flujo para ilustrar la trayectoria que seguirían los requerimientos dentro de la firma para poder ser tramitados y evacuados.

No se realizaron nuevos diagramas para el escenario 3 puesto que en este se sugirió la repartición de tareas de los abogados a otros miembros del equipo, es decir, reasignar las cargas de actividades varias no facturables, según se expuso con anterioridad. La responsabilidad de la ejecución de los trabajos facturables, que son aquellos que responden directamente los requerimientos de los clientes, sigue siendo de los abogados.

Luego de lo anterior, se hicieron las simulaciones correspondientes a partir del diagrama unificado con el programa Bizagi, tanto de los procesos actuales como de los propuestos para los escenarios 1 y 2, para poder así comparar a partir de los resultados obtenidos y determinar un estimado de liberación de tiempo de los abogados (metodología AS IS / TO BE), si lo hubiere.

Ahora bien, con el fin de que las comparaciones a realizar sean lo más ajustadas posible a la realidad, se establecieron una serie de parámetros fijos para todas las simulaciones, las cuales se indican a continuación:

- Duración: Se estableció un límite de 20 días hábiles para la simulación, siendo este el tiempo promedio de días hábiles disponibles por mes.

- Número de llegadas (requerimientos): Se calculó un estimado de 10 requerimientos diarios, para un total de 200 llegadas en los 20 días.

- Intervalo de llegadas: 60 minutos, es decir, que llegue un requerimiento cada hora por día, en una jornada laboral de 7:00 a. m. a 5:00 p. m.

- Tiempo de elaboración de respuesta a requerimiento: Se estimó un promedio de 3 horas, es decir, el tiempo total estimado que invertiría un abogado en investigación, redacción y finalización de un documento (memorial, solicitud, propuesta, concepto y demás).

- Tiempo de revisión de respuesta a requerimiento: Se estimó un promedio de 20 minutos que invertiría el abogado de nivel inmediatamente anterior en revisar cada documento.

- Tiempo de envío a corrección de la respuesta a requerimiento: Se estimó un promedio de 10 minutos que invertiría el abogado de nivel inmediatamente anterior en enviar la instrucción sobre la corrección de cada documento.

- Porcentaje de aprobación del trabajo realizado para la situación actual: Se estimó un porcentaje del $50 \%$, es decir, se espera que el $50 \%$ del trabajo realizado requiera ser corregido. 
- Porcentaje de aprobación del trabajo realizado para los escenarios 1 y 2: Se estimó un porcentaje del $80 \%$, es decir, se espera que solo el $20 \%$ del trabajo realizado requiera ser corregido.

Bizagi ofrece la posibilidad de simular los procesos con cuatro niveles, en cada uno de los cuales se puede ingresar información que va permitiendo obtener resultados más complejos, lo que permite una mayor aproximación a la realidad. Si bien dichos niveles no son restrictivos entre sí, para el desarrollo del ejercicio se corrió la simulación pasando por cada uno de ellos en el estricto orden, con el fin de ir mostrando los resultados más significativos [11],[12].

Estos niveles son:

Validación del proceso: Permite determinar si todas las actividades y caminos del proceso se cumplen correctamente. Muestra resultados en una situación ideal, es decir, sin demoras, con todos los recursos requeridos, arrojando los resultados ideales que podría alcanzar el proceso.

Análisis de tiempo: Permite conocer los tiempos de realización de cada actividad, así como el tiempo promedio y el total del procesamiento de los requerimientos recibidos dentro de los días de simulación previamente establecidos. En este punto es posible encontrar resultados un poco más ajustados a la realidad.

Análisis de recursos: Permite predecir el posible comportamiento del proceso con la utilización de determinados recursos, para el caso en particular, con la disponibilidad de distintos actores que ejecuten las diferentes actividades. Como resultado se obtendrá un porcentaje de utilización de los recursos puesto que será necesario definir cuáles son los mismos, cuántos hay disponibles y en qué actividades intervienen.

Análisis de calendarios: $\mathrm{Al}$ incluir información específica de calendarios (turnos, días hábiles, horarios, semanas y demás), permite mostrar el rendimiento del proceso según la disponibilidad de los recursos en un periodo dado de tiempo y con horarios establecidos. Para el desarrollo de este ejercicio no se trabajó esta opción, puesto que se establecieron con anterioridad un total de 20 días hábiles al mes, considerando que están incluidas la cantidad de horas diarias disponibles por cada empleado; esto, teniendo en cuenta que la empresa no maneja turnos con horarios fijos.

En relación con las simulaciones, a continuación se muestran y explican los resultados obtenidos en la de la situación actual.

\section{Validación del proceso}

En las Fig. 12 y 13 se muestran pantallazos de los resultados obtenidos en la simulación.

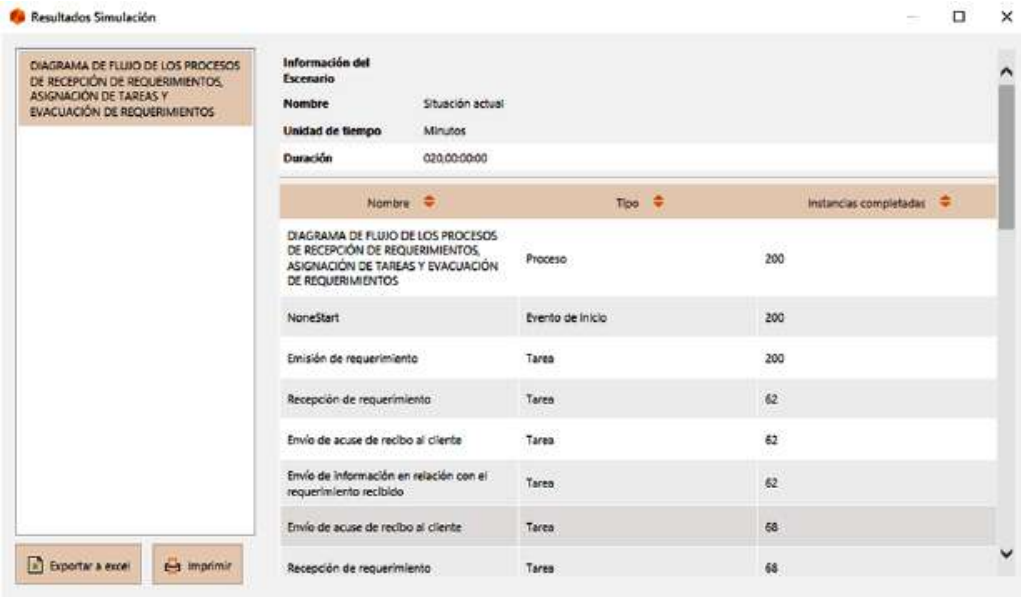

Fig. 12. Primera muestra de los resultados de la simulación por validación de la situación actual de Velasco Ordóñez S.A.S. Fuente: Los autores, basada en la información de Velasco Ordóñez S.A.S., 2019

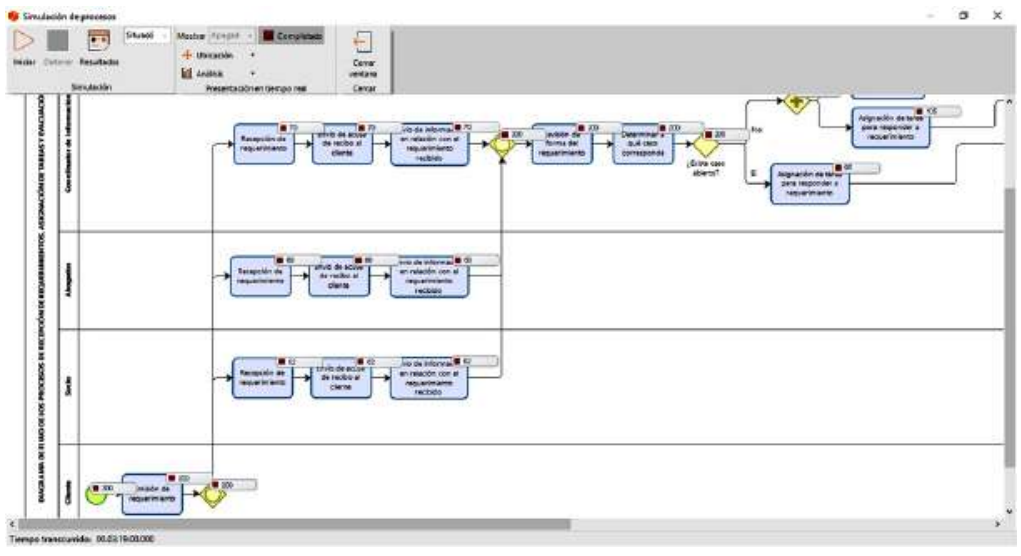

Fig. 13. Segunda muestra de los resultados de la simulación por validación de la situación actual de Velasco Ordóñez S.A.S. Fuente: Los autores, basada en la información de Velasco Ordóñez S.A.S., 2019 
La validación del proceso de la situación actual muestra que, si bien se reciben y completan 200 requerimientos, a lo largo del proceso se puede notar que la cantidad de tareas o actividades relacionadas con "responder requerimiento" dobla la de las recibidas inicialmente. Esto se debe a que se necesitan realizar correcciones al trabajo presentado la primera vez, con lo cual el proceso de elaboración de respuesta se está ejecutando con más de una vuelta, es decir, el programa estima más de una corrección por cada solicitud recibida.

\section{Análisis de tiempo}
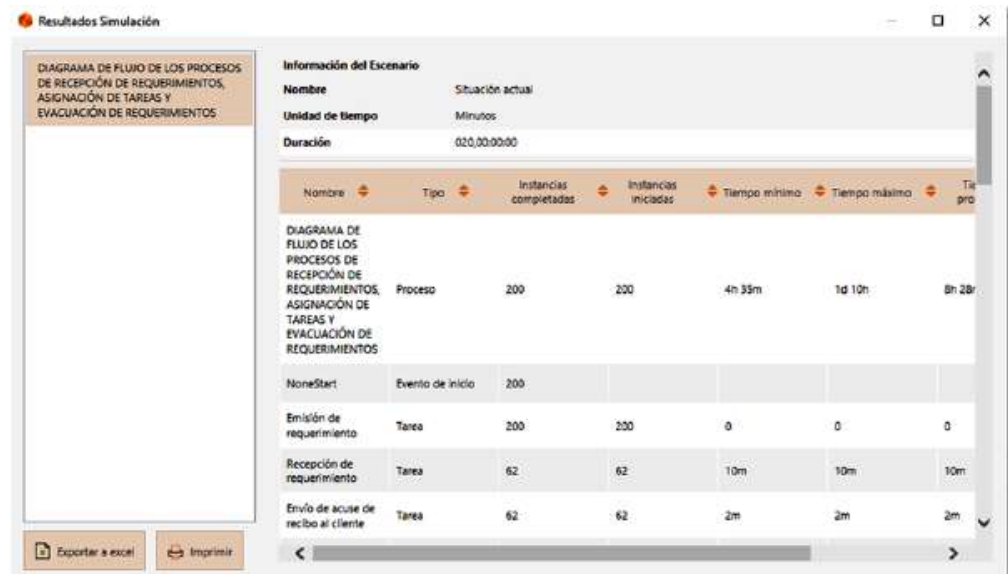

Fig. 14. Primera muestra de los resultados de la simulación por análisis de tiempo de la situación actual de Velasco Ordóñez S.A.S. Fuente: Los autores, basada en la información de Velasco Ordóñez S.A.S., 2019

En las Fig. 14 y 15 se muestran pantallazos de los resultados obtenidos en la simulación.

Los resultados indican que, en una situación ideal, el tiempo total para dar respuesta a los 200 requerimientos sería de 70 días, 17 horas y 10 minutos, es decir, tres veces más del asignado para la simulación que fue de 20 días. Asimismo se puede notar que lo mínimo que podría tardarse en dar respuesta a una solicitud serían 4 horas y 35 minutos y lo máximo sería de 1 día y 10 horas, esto es, con todas las correcciones que podrían llegar a ser tres. Finalmente, para recibir la respuesta un cliente debe esperar en promedio 8 horas y 28 minutos.

\section{Análisis de recursos}

En las Fig. 16 y 17 se muestran pantallazos de los resultados obtenidos en la simulación.

A partir de la información obtenida en las simulaciones, se realizaron las tablas IV, V y VI para la presentación de los resultados más relevantes en cuanto a uso de los recursos y tiempos de procesamiento de los requerimientos recibidos, tanto para la situación actual como para los escenarios 1 y 2.

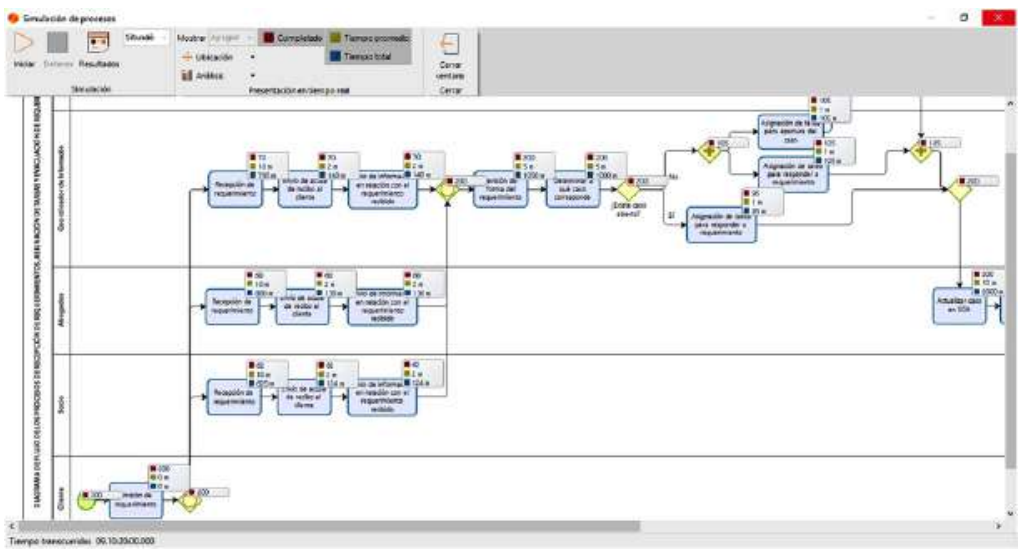

Fig. 15. Segunda muestra de los resultados de la simulación por análisis de tiempo de la situación actual de Velasco Ordóñez S.A.S. Fuente: Los autores, basada en la información de Velasco Ordóñez S.A.S., 2019

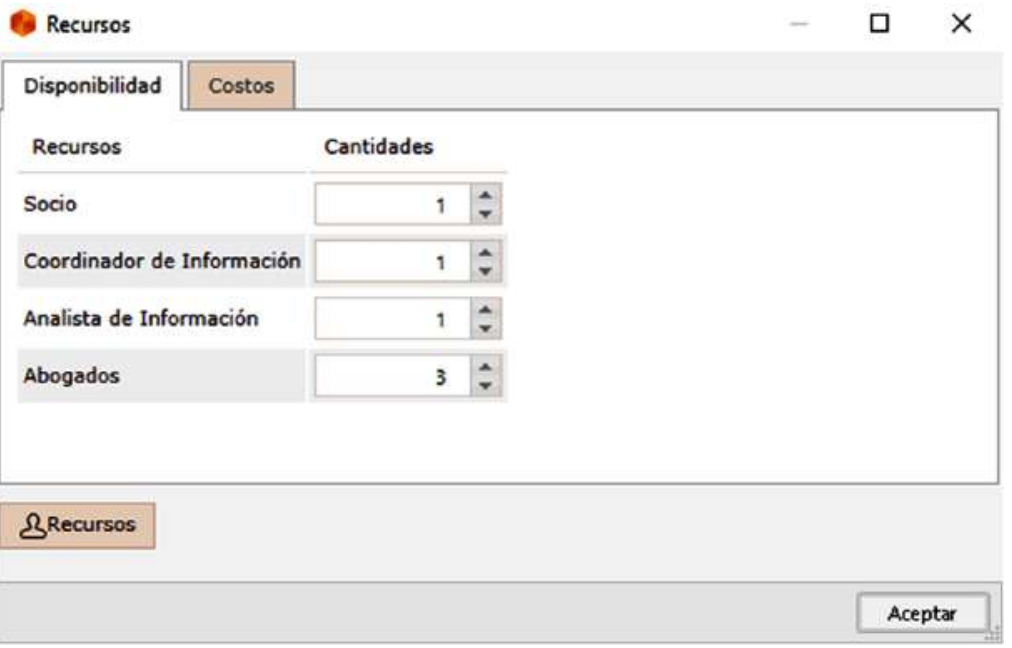

Fig. 16. Primera muestra de los resultados de la simulación por análisis de recursos de la situación actual de Velasco Ordóñez S.A.S. Fuente: Los autores, basada en la información de Velasco Ordóñez S.A.S., 2019 


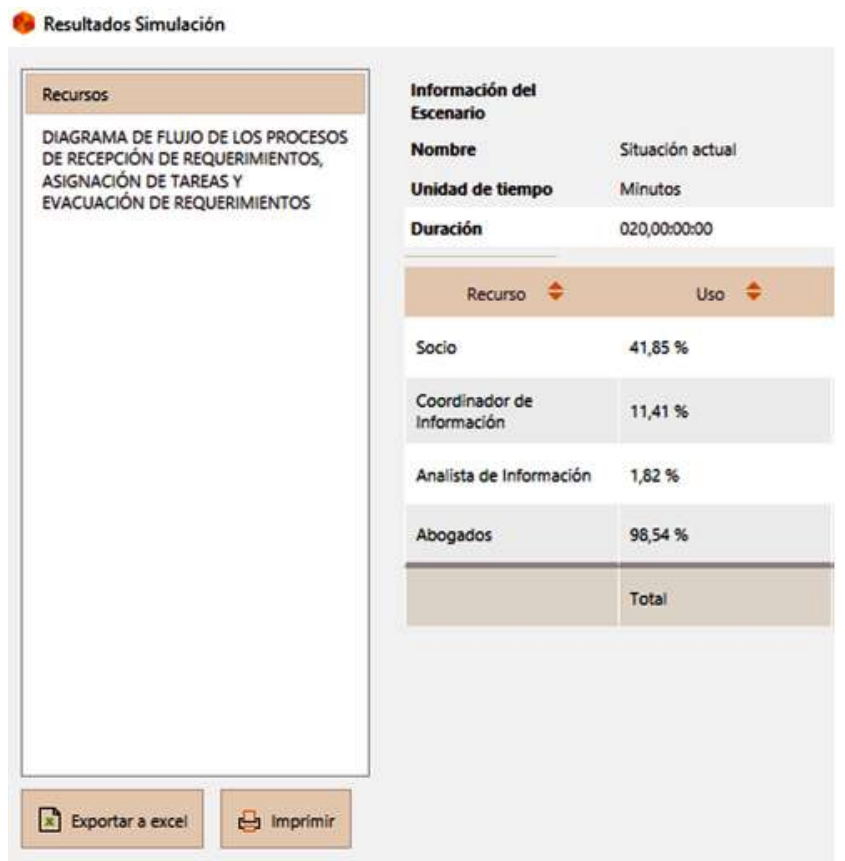

Fig. 17. Segunda muestra de los resultados de la simulación por análisis de recursos de la situación actual de Velasco Ordóñez S.A.S. Fuente: Los autores, basada en la información de Velasco Ordóñez S.A.S., 2019

Tabla V. Cantidad de respuestas enviadas a los clientes y procesos completados, a partir de la simulación por análisis de recursos.

\begin{tabular}{|l|c|c|c|c|}
\hline \multirow{2}{*}{ Análisis } & \multicolumn{3}{|c|}{$\begin{array}{c}\text { Respuestas a requerimientos } \\
\text { enviadas al cliente }\end{array}$} & \multirow{2}{*}{$\begin{array}{c}\text { Procesos } \\
\text { Nompletados }\end{array}$} \\
\cline { 2 - 4 } & Validación & $\begin{array}{c}\text { Tiemp } \\
\text { o }\end{array}$ & Recursos & \\
\hline $\begin{array}{l}\text { Situación } \\
\text { actual }\end{array}$ & 200 & 200 & 199 & 199 \\
\hline Escenario 1 & 200 & 200 & 200 & 200 \\
\hline Escenario 2 & 200 & 200 & 200 & 200 \\
\hline \multicolumn{4}{|c|}{ Fuente: Los autores, 2019 } \\
\hline
\end{tabular}

Tabla VI. Tiempos de procesamiento de los requerimientos de clientes, a partir de la simulación por análisis de recursos.

\begin{tabular}{|l|c|c|c|c|}
\hline \multicolumn{1}{|c|}{ Análisis } & $\begin{array}{c}\text { Tiempo } \\
\text { mínimo } \\
\text { (min) }\end{array}$ & $\begin{array}{c}\text { Tiempo } \\
\text { máximo } \\
\text { (min) }\end{array}$ & $\begin{array}{c}\text { Tiempo } \\
\text { promedio } \\
\text { (min) }\end{array}$ & $\begin{array}{c}\text { Tiempo } \\
\text { total(min) }\end{array}$ \\
\hline $\begin{array}{l}\text { Situación } \\
\text { actual }\end{array}$ & 450 & 24.694 & 13.637 & 2.731 .129 \\
\hline Escenario 1 & 315 & 16.037 & 6.284 & 1.283 .966 \\
\hline Escenario 2 & 259 & 33.371 & 14.144 & 2.829 .448 \\
\hline
\end{tabular}

Fuente: Los autores, 2019

Ahora bien, en la tabla VII se muestra la diferencia entre los tiempos arrojados para la situación actual y los de cada escenario, según lo presentado en la tabla VI.
Tabla VII. Diferencia entre tiempos de la situación actual y tiempos de los escenarios 1 y 2 , en minuto.

\begin{tabular}{|c|c|c|c|c|}
\hline Análisis & $\begin{array}{c}\text { Tiempo } \\
\text { mínimo } \\
\text { (min) }\end{array}$ & $\begin{array}{c}\text { Tiempo } \\
\text { máximo } \\
\text { (min) }\end{array}$ & $\begin{array}{c}\text { Tiempo } \\
\text { promedio } \\
\text { (min) }\end{array}$ & $\begin{array}{c}\text { Tiempo } \\
\text { total } \\
\text { (min) }\end{array}$ \\
\hline Escenario 1 & 135 & 8.657 & 7.352 & 1.447 .163 \\
\hline Escenario 2 & 56 & -17.334 & -7.860 & -1.545 .482 \\
\hline
\end{tabular}

Fuente: Los autores, 2019

A partir de la información obtenida en el desarrollo de los tres escenarios propuestos, se calcularon los indicadores.

Rendimiento $=$ Tiempo de respuesta $/$ requerimiento

Porcentaje de Cumplimiento $=$ Tareas asignadas/tareas realizadas a tiempo*100

Los resultados se muestran en la tabla VIII:

Tabla VIII. Rendimiento por cada escenario.

\begin{tabular}{|c|c|c|}
\cline { 2 - 3 } \multicolumn{1}{c|}{} & Rendimiento & $\begin{array}{c}\text { Porcentaje de } \\
\text { cumplimiento }\end{array}$ \\
\hline Situación actual & 228 & $99,5 \%$ \\
\hline Escenario 1 & 107 & $100,0 \%$ \\
\hline Escenario 2 & 236 & $100,0 \%$ \\
\hline Escenario 3 & 228 & $99,5 \%$ \\
\hline
\end{tabular}

Fuente: Los autores, 2019

Teniendo en cuenta que en el escenario 3 se planteó la redistribución de tareas de los abogados relacionadas con actividades no facturables, si bien se determinó una liberación de tiempo del 32,33\%, se dejaron los cálculos arrojados para la situación actual. Al respecto, es importante aclarar que esta liberación de tiempo favorece el funcionamiento de los procesos de apoyo.

\section{Evaluación ECONÓMICA}

Para la evaluación económica de los escenarios propuestos, se realizó un flujo de caja para cada uno de ellos y la situación actual. Este flujo se elaboró teniendo en cuenta:

- Se realizó un cálculo proyectado a 12 meses.

- Los comportamientos de facturación de la empresa fueron tomados como base para lo estimado.

Rev. Ingeniería, Matemáticas y Ciencias de la Información Vol. 7 / Núm. 13 / enero - junio de 2020; pág. 25-44 
- Los costos por nómina se calcularon en su totalidad, teniendo en cuenta los salarios contemplados en la situación actual, es decir, a cierre de 2018. Estos costos incluyen el personal administrativo, para una mayor aproximación a la realidad.

- Se incluye un estimado de costos administrativos y de funcionamiento.

- Los impuestos calculados corresponden a los que están relacionados con la actividad de la empresa.

- El flujo elaborado para la situación correspondería al mismo del escenario 3, puesto que en este no se plantea inversión por contratación de personal ni de tecnología.

Ahora, si bien los resultados no evidencian pérdidas, si se refleja una disminución en la utilidad para los escenarios 1 y 2 . Al respecto es importante tener en cuenta que la liberación del uso de tiempo de los abogados y del socio permitiría a la empresa no solo concretar nuevos negocios, sino también aumentar la base de facturación. En relación con esto, no se estimó un incremento en las ventas mensuales puesto que se realizó el ejercicio teniendo en cuenta las políticas de la compañía.

De esta manera su sugiere considerar qué escenario permite no solo una liberación de tiempo a los abogados sino también cuál de ellos ayuda a dar respuesta a los requerimientos en un menor tiempo, aumentado la capacidad de atención de la compañía. Por ser una empresa prestadora de servicios relacionados con consultoría jurídica, su recurso más valioso es el tiempo invertido por cada abogado y la oportunidad que se le pueda brindar a los clientes.

\section{Conclusiones}

La metodología BPM permitió no solo la detección de variables influyentes para el desarrollo del proceso sino que también proporcionó herramientas que permitan a la empresa analizar la situación actual, buscar posibles caminos a seguir y, finalmente, tener información que le permita tomar decisiones en pro de la mejora continua.
Debido a que el tiempo es el factor que más incide en la productividad de la empresa y teniendo en cuenta que las simulaciones demostraron que los diferentes procesos alteran los tiempos mínimos, máximos y promedio, el seguimiento constante de los procesos permitirá a la empresa determinar en qué se puede mejorar el uso del tiempo y aumentar su nivel de productividad.

Por otro lado, los escenarios propuestos estiman que haya una reducción de tiempo considerable para los abogados, lo que le permitirá a la empresa no solo cumplir a tiempo con los compromisos adquiridos con los clientes sino también estructurar un sistema de atención de requerimientos extraordinarios para no afectar los flujos normales de los procesos.

\section{Discusión}

El proyecto "Estructuración del proceso de abastecimiento de suministros y comercialización de productos para Dicol Ltda. mediante la metodología de Busines Process Management (BPM)" permitió el rediseño de los procesos de compras, sala de ventas y asesores comerciales, y comercio exterior, se obtuvo una mejora en los tiempos de procesamiento, lo que a su vez ayuda a incrementar la capacidad de los procesos. Por otro lado, en aporte al desarrollo de la metodología del proceso presentado en el artículo, permitió determinar con mayor precisión las variables críticas, lo cual le permite a la empresa no solo establecer posibles soluciones sino que también le ayuda a la mejora de los procesos a partir de los recursos con los que ya cuenta la empresa, es decir, le permite notar sus puntos fuertes y hacer que sean ellos mismos los que se conviertan en parte de la solución, lo cual es económicamente favorable para la misma utilizando diferentes herramientas de prospectiva para la validación de los resultados.

\section{REFERENCIAS}

[1] Bello, J.M., Uribe, C.A. y Núñez, O.F., Ciclo de Vida BPM. Recuperado de https:// bpmsosw.wordpress. com/2012/02/12/ciclo-de-vida-bpm/. 2012

[2] Garimella, K., Lees M. y Williams, B., BPM (Gerencia de Procesos de Negocio). Recuperado de http:/ /www.konradlorenz.edu.co/images / publicaciones/suma_digital_sistemas/bpm.pdf 
[3] IBM Knowledge Center (s.f.), Diagramas de procesos. Recuperado de https://www.ibm.com/ support/knowledgecenter/es/SSBJCK_7.0.0/ com.ibm.btools.modeler.basic.model.doc/model elements/processdiagram.html

[4] Morales, C.B. y Vega, M.A., Estructuración del proceso de abastecimiento de suministros y comercialización de productos para dicol ltda mediante la metodología business process management (BPM). Recuperado de http:/ / repository.unilibre. edu.co/bitstream/handle/10901/7850/Morales ArevaloCristianBenigno2015.pdf? sequence $=$ 1\&isAllowed=y. 2014

[5] Morales, C.B. y Vega, M.A., La Matriz FODA Cruzada para ideas de negocios. Recuperado de http:/ /gestionando-empresas.blogspot.com/2010/08/ la-matriz-foda-cruzada-para-ideas-de.html. 2014

[6] Gestionando-empresas.blogspot.com, Estructuración del proceso de abastecimiento de suministros y comercialización de productos para dicol ltda mediante la metodología business process management (BPM). Recuperado de http:/ / gestionandoempresas.blogspot.com/2010/08/la-matriz-fodacruzada-para-ideas-de.html. 2014
[7] Planeacionestrategica.blogspot.es, Matriz EFEEFI - Planeacion Estrategica. Recuperado de http: / / planeacionestrategica.blogspot.es/124389 7868/matriz-efe-efi/

[8] Hay una luz que nunca se apaga, Matriz IGO 2013. Recuperado de https:/ / ramonchung.wordpress. com/2013/02/28/matriz-igo/. 2013

[9] encolombia.com, Variables Tecnológicas de Cadena Productiva Algodón. Recuperado de https:/ / encolombia.com/economia/info-economica/ algodon/identificaciondelos factores1/

[10] Senaprevios.com, SENA PREVIOS. Recuperado de http://www.senaprevios.com/wp-content/ uploads/2017/instrutivos/guia-de-metodosprospectivos.pdf. 2017

[11] HEFLO ES, Las 6 fases del ciclo de vida BPM y cómo utilizar en su empresa. Recuperado de https: //www.heflo.com/ es/blog/bpm/ciclo-vidabpm/

[12] HEFLO ES, Conozca 12 tipos de indicadores de rendimiento fundamentales. Recuperado de https: / / www.heflo.com/es/blog/planificacionestrategica/tipos-indicadores-rendimiento/ 